\title{
Computing Elementary Symmetric Functions and Their Derivatives: A Didactic
}

\author{
Frank B. Baker, University of Wisconsin \\ Michael R. Hawell, University of Pistsburgh
}

The computation of elementary symmetric functions and their derivatives is an integral part of conditional maximum likelihood estimation of item parameters under the Rasch model. The conditional approach has the advantages of parameter estimates that are consistent (assuming the model is correct) and statistically rigorous goodness-of-fit tests. Despite these characteristics, the conditional approach has been limited by problems in computing the elementary symmetric functions. The introduction of recursive formulas for computing these functions and the availability of modern computers has

\begin{abstract}
largely mediated these problems; however, detailed documentation of how these formulas work is lacking. This paper describes how various recursion formulas work and how they are used to compute elementary symmetric functions and their derivatives. The availability of this information should promote a more thorough understanding of item parameter estimation in the Rasch model among both measurement specialists and practitioners. Index terms: algorithms, computational techniques, conditional maximum likelihood, elementary symmetric functions, Rasch model.
\end{abstract}

The Rasch model is a popular item response theory model that is based on two parameters, an examinee trait parameter $\xi_{v}, 0 \leq \xi_{v} \leq \infty, v=1,2,3, \ldots, N$ and an item easiness/difficulty parameter $\varepsilon_{i}, 0 \leq \varepsilon_{i} \leq \infty, i=1$, $2,3, \ldots, k$. Given $\xi$ and $\varepsilon$, the likelihood of response $u_{i v}$ to dichotomously scored item $i$ is given by

$P\left(U_{i v}=u_{i v} \mid \xi_{v}, \varepsilon_{i}\right)=\frac{\left(\xi_{v} \varepsilon_{i}\right)^{u_{i v}}}{1+\xi_{v} \varepsilon_{i}}$,

where $u_{i v}$ represents the response of the $v$ th examinee to the ith item and is the realization of $U_{i v}\left(u_{i v}=1\right.$ if the item is answered correctly and 0 otherwise). Rasch (1960/80) modeled the combinatorial aspects of responses to dichotomously scored test items in terms of elementary symmetric functions (ESFs):

$\gamma_{r}=\gamma_{r}\left\{\left(\varepsilon_{i}\right)\right\}=\sum_{\left(u_{i}\right)=r} \prod_{i=1}^{k} \varepsilon_{i}^{u_{i n}}$

where the summation is over all vectors of item responses that contain $r$ correct responses.

Andersen (1970, 1972) used Rasch's (1960/80, 1961) work as the basis for the conditional maximum likelihood estimation (CMLE) procedure for estimating item parameters, in which the $\xi$ s are replaced by their sufficient statistics, which are their number-correct test scores, and item parameters are estimated conditionally on these statistics. The resultant likelihood function (Fischer, 1973) is given by

$l=\left[\left(\begin{array}{c}\left(f_{r}\right) \\ \left(s_{i}\right)\end{array}\right] \prod_{r=1}^{k} \gamma_{i=1}^{k} \varepsilon_{i}^{s_{r}}\right.$,

\section{APPLIED PSYCHOLOGICAL MEASUREMENT}

Vol. 20, No. 2, June 1996, pp.169-192

(C) Copyright 1996 Applied Psychological Measurement Inc.

0146-6216/96/0200169-24\$2.45 
where

$r_{v}=\sum_{i=1}^{k} u_{i v}$

is the number-correct score for examinee $v$ (when it creates no confusion a number-correct score is denoted by $r), f_{r}$ is the number of examinees with a number-correct score of $r$, and

$s_{i}=\sum_{y=1}^{N} u u_{i v}$

is the item score for item $i$. Taking the derivative of the loglikelihood with respect to an arbitrary $\varepsilon$ parameter and multiplying through by $\varepsilon_{i}$ results in

$s_{i}-\sum_{r=1}^{k} \frac{\varepsilon_{i} f_{r} \gamma_{r-1}^{(i)}}{\gamma_{r}}=0$,

where

$\gamma_{r-1}^{(i)}=\frac{\partial \gamma_{r}}{\partial \varepsilon_{i}}$

is the first derivative of the symmetric function $\gamma_{r}$ with respect to $\varepsilon_{i}$ and is an ESF of order $r-1$. The resulting system of $k$ likelihood equations can be solved using the Broyden-Fletcher-Goldfarb-Shanno or gradient methods (Press, Flannery, Teukolsky, \& Vetterling, 1986) that involve both the ESFs and their first derivatives, or using the Newton-Raphson procedure (Baker, 1992). Thus, numerical evaluation of the ESFs and their derivatives is central to CMLE under the Rasch model and its extensions.

In recent years, a number of procedures for evaluating the ESFs and their derivatives have been developed (Fischer, 1995; Fischer \& Ponocny, 1994, 1995; Formann, 1986; Gustafsson, 1980; Jansen, 1984; Verhelst, Glas, \& van der Sluis, 1984). These approaches are based on the difference algorithm (Fischer, 1974; Fischer \& Allerup, 1968), the summation algorithm (Fischer, 1974), or a combination of these two algorithms within the context of the grouping property of ESFs (Liou, 1994; Verhelst \& Veldhuijzen, 1991). Although the mathematical formulation of these algorithms and the grouping approach have been presented in the literature, the numerical details have not. The goal here was to provide both measurement specialists and practitioners an understanding of the computational aspects of ESFS as used in CMLE under the Rasch model and its extensions.

\section{Computing ESFS Using the Difference Algworithm}

A serious problem in the computation of the $\gamma_{r}$ is that the number of multiplications becomes very large as the number of items (and hence $r$ ) increases. Gustafsson (1977) reported that when the number of items is 50 and $r=25, \gamma_{25}$ is defined as the sum of $1.26 \times 10^{14}$ terms, each of which is a product of 25 terms. Even for modern computers, it is not feasible to compute the $\gamma_{r}$ for even shor tests (e.g., 15 items) using direct multiplication, without substantial rounding errors occurring. Fischer (1974, pp. 242-244) developed an algorithm that avoided direct multiplication by relying on recursion formulas for computing the ESFs and their first derivatives. Gustafsson (1977) called this the difference algorithm and indicated that it leads to less rounding error than direct multiplication and is fast for $20 \leq k \leq 40$. The description of the difference algorithm below relies heavily on the FORTRAN subroutine GAMMA found in Fischer \& Formann's (1972) LLTM computer program (which required only 41 lines of FORTRAN code) and in the PC version of the LLTM program (Fischer \& Wild, 1988), and Fischer's (1974) book. This subroutine was also used in Gustafsson's $(1977,1990)$ PML computer program.

The recursive relationship between an ESF of order $r$ and its derivative with respect to $\varepsilon$ as provided by 
Rasch (1960, p. 180 , eq. 6.10$)$ is

$\gamma_{r}=\varepsilon_{i} \gamma_{r-1}^{(i)}+\gamma_{r}^{(i)}$,

where

$\gamma_{r-1}^{(i)}=\frac{\partial \gamma_{r}}{\partial \varepsilon_{i}}$

is a symmetric function of order $r-1$ in all $k$ item parameters except $\varepsilon_{i}$. For example, if $r=2$ then

$\gamma_{1}^{(i)}=\gamma_{2-1}^{(i)}=\frac{\partial \gamma_{2}}{\partial \varepsilon_{i}}$,

and

$\gamma_{r}^{(i)}=\frac{\partial \gamma_{r+1}}{\partial \varepsilon_{i}}$

is a symmetric function of order $r$ in all $k$ item parameters except $\varepsilon_{i}$. If $r=2$, then $r+1=3$ and

$\gamma_{2}^{(i)}=\gamma_{3-1}^{(i)}=\frac{\partial \gamma_{3}}{\partial \varepsilon_{i}}$.

Whenever an ESF has a superscript, the letter within the parentheses indicates that a derivative of the next higher-order ESF has been taken with respect to $\varepsilon$, indexed by the letter. The subscript of the ESF indicates its order. For example, the subscript $r$ appears on both sides of Equation 8. On the left, the $\gamma_{r}$ is of order $r$ and involves all $k \varepsilon$ parameters; that is, $\varepsilon_{1}, \varepsilon_{2}, \ldots, \varepsilon_{k}$. On the right side, $\gamma_{1}^{(i)}$ is also of order $r$ but involves only $k-1 \varepsilon$ parameters $-\varepsilon_{1}, \varepsilon_{2}, \ldots, \varepsilon_{i-1}, \varepsilon_{i+1}, \ldots, \varepsilon_{k}$. Symbolically, if $\gamma_{1}$ and $\gamma_{1}^{(i)}$ are in an equation, both subscripts denote ESFS of order 1 but they involve item parameter sets of different size.

The recursion formula that is the basis of the difference algorithm is a simple rewrite of Equation 8:

$\gamma_{r}^{(i)}=\gamma_{r}-\varepsilon_{i} \gamma_{r-1}^{(i)}$.

The following relationship (Fischer, 1974, p. 242) also holds:

$r \gamma_{r}=\sum_{i=1}^{k} \varepsilon_{i} \gamma_{r-1}^{(i)}$.

To illustrate the difference algorithm, suppose that the $\gamma_{r}$ for a 5 -item test (Table 1 ) are to be computed. Using Equations 13 and 14, it is possible to devise an efficient algorithm for computing the $\gamma_{r}$ and their first derivatives. Table 2 shows the needed derivatives in symbolic form for $r=1,2, \ldots, 5$.

Using the difference algorithm, the GAMMA subroutine reduces the accumulation of round-off errors in the computation of the $\gamma_{r}$ by breaking the computations into low-to-high and high-to-low parts (Fischer, 1974). The low-to-high part computes $\gamma_{r}$ for $r=1,2, \ldots, k / 2$; the high-to-low part computes the symmetric functions for $r=k, k-1, k-2, \ldots, k / 2$. The midpoint value is determined by the FORTRAN rules for integer division. For example, when $k=5,5 / 2+1=2+1=3$ and if $k$ is even, say $k=4,4 / 2+1=2+1=3$, so that $\gamma_{3}$ is the midpoint in either case.

The low-to-high part is illustrated using the product-normalized values of $\varepsilon_{i}$ for the 5 items of the LSAT-6 dataset (Bock \& Lieberman, 1970) under the Rasch model, which is known to fit these data well (Thissen, 1982). The LLTM computer program (Fischer \& Formann, 1972) produced estimated $\varepsilon$ parameters of $\varepsilon_{1}=$ $3.5118, \varepsilon_{2}=.6219, \varepsilon_{3}=.2905, \varepsilon_{4}=.8450$, and $\varepsilon_{5}=1.8648$. In practice, CMLE starts by computing the ESFs using the initial estimates of the $\varepsilon_{i}$. The final values were used here to provide a link to previous results in the literature (Baker, 1992; Thissen, 1982).

The logic of the difference algorithm is based on proceeding column-wise from left to right across Table 2. 


\section{2

Toble 1

Elementary Symmetric Functions for $k=5$ items

\begin{tabular}{lll}
\hline \hline \multicolumn{1}{c}{$\gamma_{r}$} & $\left(\begin{array}{l}k \\
r\end{array}\right)$ \\
\hline $0 \quad \gamma_{0}=1$ & $\left(\begin{array}{l}5 \\
0\end{array}\right)=1$ \\
$1 \quad 1 \gamma_{1}=\varepsilon_{1}+\varepsilon_{2}+\varepsilon_{3}+\varepsilon_{4}+\varepsilon_{5}$ & $\left(\begin{array}{l}5 \\
1\end{array}\right)=5$ \\
$2 \quad \gamma_{2}=\varepsilon_{1} \varepsilon_{2}+\varepsilon_{1} \varepsilon_{3}+\varepsilon_{1} \varepsilon_{4}+\varepsilon_{1} \varepsilon_{5}+\varepsilon_{2} \varepsilon_{3}+\varepsilon_{2} \varepsilon_{4}+\varepsilon_{2} \varepsilon_{5}+\varepsilon_{3} \varepsilon_{4}+\varepsilon_{3} \varepsilon_{5}+\varepsilon_{4} \varepsilon_{5}$ & $\left(\begin{array}{l}5 \\
2\end{array}\right)=10$ \\
$3 \quad \gamma_{3}=\varepsilon_{1} \varepsilon_{2} \varepsilon_{3}+\varepsilon_{1} \varepsilon_{2} \varepsilon_{4}+\varepsilon_{1} \varepsilon_{2} \varepsilon_{5}+\varepsilon_{1} \varepsilon_{3} \varepsilon_{4}+\varepsilon_{1} \varepsilon_{3} \varepsilon_{5}+\varepsilon_{1} \varepsilon_{4} \varepsilon_{5}+\varepsilon_{2} \varepsilon_{3} \varepsilon_{4}+\varepsilon_{2} \varepsilon_{3} \varepsilon_{5}+\varepsilon_{2} \varepsilon_{4} \varepsilon_{5}+\varepsilon_{3} \varepsilon_{4} \varepsilon_{5}$ & $\left(\begin{array}{l}5 \\
3\end{array}\right)=10$ \\
$4 \quad \gamma_{4}=\varepsilon_{2} \varepsilon_{3} \varepsilon_{4} \varepsilon_{5}+\varepsilon_{1} \varepsilon_{3} \varepsilon_{4} \varepsilon_{5}+\varepsilon_{1} \varepsilon_{2} \varepsilon_{4} \varepsilon_{5}+\varepsilon_{1} \varepsilon_{2} \varepsilon_{3} \varepsilon_{5}+\varepsilon_{1} \varepsilon_{2} \varepsilon_{3} \varepsilon_{4}$ & $\left(\begin{array}{l}5 \\
4\end{array}\right)=5$ \\
5 & $\gamma_{5}=\varepsilon_{1} \varepsilon_{2} \varepsilon_{3} \varepsilon_{4} \varepsilon_{5}$ & $\left(\begin{array}{l}5 \\
5\end{array}\right)=1$ \\
\hline
\end{tabular}

The process begins by computing the lowest-order ESF $(r=1)$ and its first derivatives with respect to each of the $k \varepsilon$ parameters. Then Equation 13 is used to obtain the first derivatives of the next higher-order ESF, and the value of the ESF is obtained using Equation 14. The order of the ESF is increased by 1 and the process is repeated until the highest-order ESF has been computed. The end products are the numerical values of the $\gamma_{r}$ $(r=1,2, \ldots, k)$ and $\gamma_{r-1}^{(i)}(r-1=0,1,2, \ldots, k-1)$, which are reported in Table 3 for the LSAT 6 data.

Table 2

First Derivatives of the $\gamma_{r}$ and $r-1$

\begin{tabular}{cccccc}
\multicolumn{7}{c}{ First Derivatives of the $\gamma_{r}$ and $r-1$} \\
\cline { 2 - 6 } & \multicolumn{7}{c}{$r$ (Top Row) and $r$ - } & (Bottom Row) \\
\hline$\varepsilon_{i}$ & 0 & 2 & 3 & 4 & 5 \\
\hline$\varepsilon_{1}$ & $\gamma_{0}^{(1)}$ & $\gamma_{1}^{(1)}$ & $\gamma_{2}^{(1)}$ & $\gamma_{3}^{(1)}$ & $\gamma_{4}^{(1)}$ \\
$\varepsilon_{2}$ & $\gamma_{0}^{(2)}$ & $\gamma_{1}^{(2)}$ & $\gamma_{2}^{(2)}$ & $\gamma_{3}^{(2)}$ & $\gamma_{4}^{(2)}$ \\
$\varepsilon_{3}$ & $\gamma_{0}^{(3)}$ & $\gamma_{1}^{(3)}$ & $\gamma_{2}^{(3)}$ & $\gamma_{3}^{(3)}$ & $\gamma_{4}^{(3)}$ \\
$\varepsilon_{4}$ & $\gamma_{0}^{(4)}$ & $\gamma_{1}^{(4)}$ & $\gamma_{2}^{(4)}$ & $\gamma_{3}^{(4)}$ & $\gamma_{4}^{(4)}$ \\
$\varepsilon_{5}$ & $\gamma_{0}^{(5)}$ & $\gamma_{1}^{(5)}$ & $\gamma_{2}^{(5)}$ & $\gamma_{3}^{(5)}$ & $\gamma_{4}^{(5)}$ \\
$\sum_{i=1}^{k} \varepsilon_{i} \gamma_{r-1}^{(i)}$ & $1 \gamma_{1}$ & $2 \gamma_{2}$ & $3 \gamma_{3}$ & $4 \gamma_{4}$ & $5 \gamma_{5}$ \\
\hline
\end{tabular}

Implementation of the difference algorithm begins at the $r=1$ column, where the $\gamma_{r-1}^{(i)}=\gamma_{0}^{(i)}$ need to be computed. Table 1 shows that $\gamma_{1}$ is the sum of the five $\varepsilon$ parameters, and that the derivative of this quantity with respect to any $\varepsilon_{i}$ will be $1\left[\gamma_{0}^{(i)}=1\right.$ for $\left.i=1,2, \ldots, 5\right]$. Hence, the numerical values for all entries in the $r-1=0$ column of Table 2 will be 1 . To obtain the marginal value for column 1 , the sum of the products of $\varepsilon_{i}$ and $\gamma_{0}^{(i)}$ (i.e., the inner product), where $i=1,2, \ldots, 5$, is computed. Table 3 shows that this sum is 
$\sum_{i=1}^{5} \varepsilon_{i} \gamma_{0}^{(i)}=(1) \varepsilon_{1}+(1) \varepsilon_{2}+(1) \varepsilon_{3}+(1) \varepsilon_{4}+(1) \varepsilon_{5}=7.1340$

Using Equation 14 with $r=\mathbb{1}$,

$\gamma_{1}=\frac{\sum_{i}^{5} \varepsilon_{i} \gamma_{0}^{(i)}}{1}=7.1340$.

Next, the entries in the second column of Table 2 [the $\gamma_{2-1}^{(i)}$ terms] are needed to obtain $\gamma_{2}$ and are computed using Equation 13:

$\gamma_{1}^{(1)}=\gamma_{1}-\varepsilon_{1} \gamma_{0}^{(1)}=\varepsilon_{1}+\varepsilon_{2}+\varepsilon_{3}+\varepsilon_{4}+\varepsilon_{5}-\varepsilon_{1}(1)=\varepsilon_{2}+\varepsilon_{3}+\varepsilon_{4}+\varepsilon_{5}=3.6222$

$\gamma_{1}^{(2)}=\gamma_{1}-\varepsilon_{2} \gamma_{0}^{(2)}=\varepsilon_{1}+\varepsilon_{2}+\varepsilon_{3}+\varepsilon_{4}+\varepsilon_{5}-\varepsilon_{2}(1)=\varepsilon_{1}+\varepsilon_{3}+\varepsilon_{4}+\varepsilon_{5}=6.5121$

$\gamma_{1}^{(3)}=\gamma_{1}-\varepsilon_{3} \gamma_{0}^{(3)}=\varepsilon_{1}+\varepsilon_{2}+\varepsilon_{3}+\varepsilon_{4}+\varepsilon_{5}-\varepsilon_{3}(1)=\varepsilon_{1}+\varepsilon_{2}+\varepsilon_{4}+\varepsilon_{5}=6.8435$

$\gamma_{1}^{(4)}=\gamma_{1}-\varepsilon_{4} \gamma_{0}^{(4)}=\varepsilon_{1}+\varepsilon_{2}+\varepsilon_{3}+\varepsilon_{4}+\varepsilon_{5}-\varepsilon_{4}(1)=\varepsilon_{1}+\varepsilon_{2}+\varepsilon_{3}+\varepsilon_{5}=6.2890$

$\gamma_{1}^{(5)}=\gamma_{1}-\varepsilon_{5} \gamma_{0}^{(5)}=\varepsilon_{1}+\varepsilon_{2}+\varepsilon_{3}+\varepsilon_{4}+\varepsilon_{5}-\varepsilon_{5}(1)=\varepsilon_{1}+\varepsilon_{2}+\varepsilon_{3}+\varepsilon_{4}=5.2692$.

The marginal value for this column is the sum of the products of $\varepsilon_{i}$ and $\gamma_{1}^{(i)}$ :

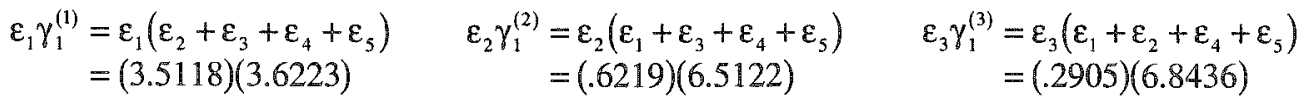

$$
\begin{aligned}
& =12.7204 \quad=4.0499 \quad=1.9880 \\
& \begin{aligned}
\varepsilon_{4} \gamma_{1}^{(4)} & =\varepsilon_{4}\left(\varepsilon_{1}+\varepsilon_{2}+\varepsilon_{3}+\varepsilon_{5}\right) & \varepsilon_{5} \gamma_{1}^{(5)} & =\varepsilon_{5}\left(\varepsilon_{1}+\varepsilon_{2}+\varepsilon_{3}+\varepsilon_{4}\right) \\
& =(.8450)(6.2891) & & =(1.8648)(5.2693) \\
& =5.3142 & & =9.8260
\end{aligned} \quad \sum_{i=1}^{k} \varepsilon_{i} \gamma_{1}^{(i)}=33.8986 .
\end{aligned}
$$

Note that the product of each pair of item parameters appears twice in the total. From Equation 14 it is known that

$2 \gamma_{2}=\sum_{i=1}^{5} \varepsilon_{i} \gamma_{1}^{(i)}$

thus,

$\gamma_{2}=\frac{33.8986}{2}=16.9493$

Table 3

\begin{tabular}{|c|c|c|c|c|c|}
\hline & & $r$ (Top Ro & and $r-1$ (Bott & Row) & \\
\hline & 1 & 2 & 3 & 4 & 5 \\
\hline$\varepsilon_{i}$ & 0 & 1 & 2 & 3 & 4 \\
\hline 3.5118 & 1 & 3.6222 & 4.2288 & 1.9273 & .2847 \\
\hline .6219 & 1 & 6.5121 & 12.8994 & 8.7560 & 1.6076 \\
\hline .2905 & 1 & 6.8435 & 14.9612 & 12.4319 & 3.4414 \\
\hline .8450 & $\mathbb{1}$ & 6.2890 & 11.6351 & 6.9465 & 1.1831 \\
\hline 1.8648 & 1 & 5.2692 & 7.1233 & 3.4946 & .5361 \\
\hline$\sum_{i=1}^{5} \varepsilon_{i} \gamma_{r-1}^{(i)}$ & 7.1340 & 33.8986 & 50.3343 & 33.5562 & 7.0529 \\
\hline & $\gamma_{1}=7.1340$ & $\gamma_{2}=\mathbb{1} 6.9493$ & $\gamma_{3}=16.7781$ & $\gamma_{4}=7.0529$ & $\gamma_{5}=.9997$ \\
\hline
\end{tabular}

First Derivatives $\gamma_{r-1}^{(i)}$ and Values of $\gamma_{r}$

Downloaded from the Digital Conservancy at the University of Minnesota, http://purl.umn.edu/93227. 


\section{APPLIED PSYCHOLOGICAL MEASUREMENT}

Next, compute the $\gamma_{2}^{(i)}$ entries for the $r-1=2$ column of Table 2, which in turn allows $\gamma_{3}$ to be computed. As before, use Equation 13 to compute

$\gamma_{3-1}^{(i)}=\frac{\partial \gamma_{3}}{\partial \varepsilon_{i}}=\gamma_{2}^{(i)}$.

For $i=1$,

$$
\begin{aligned}
\gamma_{2}^{(1)}=\gamma_{2}-\varepsilon_{1} \gamma_{1}^{(1)}=\varepsilon_{1} \varepsilon_{2}+\varepsilon_{1} \varepsilon_{3}+\varepsilon_{1} \varepsilon_{4}+\varepsilon_{1} \varepsilon_{5} & +\varepsilon_{2} \varepsilon_{3}+\varepsilon_{2} \varepsilon_{4}+\varepsilon_{2} \varepsilon_{5}+\varepsilon_{3} \varepsilon_{4}+\varepsilon_{3} \varepsilon_{5}+\varepsilon_{4} \varepsilon_{5}-\varepsilon_{1}\left(\varepsilon_{2}+\varepsilon_{3}+\varepsilon_{4}+\varepsilon_{5}\right) \\
=\varepsilon_{2} \varepsilon_{3}+\varepsilon_{2} \varepsilon_{4}+\varepsilon_{2} \varepsilon_{5}+\varepsilon_{3} \varepsilon_{4}+\varepsilon_{3} \varepsilon_{5}+\varepsilon_{4} \varepsilon_{5} & =16.9493-3.5118(3.6222) \\
& =16.9493-12.7204 \\
& =4.2288 .
\end{aligned}
$$

This is an ESF of order 2 involving $k=5-1$ elements, namely: $\varepsilon_{2}, \varepsilon_{3}, \varepsilon_{4}$, and $\varepsilon_{5}$. Then,

$$
\begin{aligned}
\gamma_{2}^{(2)}=\gamma_{2}-\varepsilon_{2} \gamma_{1}^{(2)}=\varepsilon_{1} \varepsilon_{3}+\varepsilon_{1} \varepsilon_{4}+\varepsilon_{1} \varepsilon_{5}+\varepsilon_{3} \varepsilon_{4}+\varepsilon_{3} \varepsilon_{5}+\varepsilon_{4} \varepsilon_{5} & =16.9493-.6219(6.5121) \\
& =16.9493-4.0499=12.8994 \\
\gamma_{2}^{(3)}=\gamma_{2}-\varepsilon_{3} \gamma_{1}^{(3)}=\varepsilon_{1} \varepsilon_{2}+\varepsilon_{1} \varepsilon_{4}+\varepsilon_{1} \varepsilon_{5}+\varepsilon_{2} \varepsilon_{4}+\varepsilon_{2} \varepsilon_{5}+\varepsilon_{4} \varepsilon_{5} & =16.9493-.2905(6.8435) \\
& =16.9493-1.9881=14.9612 \\
\gamma_{2}^{(4)}=\gamma_{2}-\varepsilon_{4} \gamma_{1}^{(4)}=\varepsilon_{1} \varepsilon_{2}+\varepsilon_{1} \varepsilon_{3}+\varepsilon_{1} \varepsilon_{5}+\varepsilon_{2} \varepsilon_{3}+\varepsilon_{2} \varepsilon_{5}+\varepsilon_{3} \varepsilon_{5} & =16.9493-.8450(6.2896) \\
& =16.9493-5.3142=11.6351 \\
\gamma_{2}^{(5)}=\gamma_{2}-\varepsilon_{5} \gamma_{1}^{(5)}=\varepsilon_{1} \varepsilon_{2}+\varepsilon_{1} \varepsilon_{3}+\varepsilon_{1} \varepsilon_{4}+\varepsilon_{2} \varepsilon_{3}+\varepsilon_{2} \varepsilon_{4}+\varepsilon_{3} \varepsilon_{4} & =16.9493-1.8648(5.2692) \\
& =16.9493-9.8260=7.1233
\end{aligned}
$$

The column marginal is computed using the inner product of the vectors $\varepsilon$ and $\gamma_{2}=(i)$, yielding

$$
\sum_{i=1}^{5} \varepsilon_{i} \gamma_{2}^{(i)}=50.3343
$$

Note that each pair appears three times in the final sum. Then from Equation 14:

$$
\gamma_{3}=\frac{\sum_{i=1}^{5} \varepsilon_{i} \gamma_{2}^{(i)}}{3}=\frac{50.3343}{3}=16.7781 \text {. }
$$

The upper limit of the low-to-high part of the process is reached at $r=3$, and the values in the first three columns of Table 3 have been filled. The paradigm now shifts to the high-to-low part to fill in the remaining cells in Table 3.

The high-to-low part is based on a rewriting of Equation 13 (Fischer, 1974):

$$
\gamma_{r-1}^{(i)}=\left[\gamma_{r}-\gamma_{r}^{(i)}\right] / \varepsilon_{i} \text {. }
$$

Fischer (1974, p. 243) provided the following relationship in place of Equation 14 for use in the high-tolow part:

$(k-r) \gamma_{r}=\sum_{i=1}^{k} \gamma_{r}^{(i)}$

The high-to-low part begins by computing $\gamma_{5}$, which is $\gamma_{5}=\varepsilon_{1} \varepsilon_{2} \varepsilon_{3} \varepsilon_{4} \varepsilon_{5}=1.0$. In general, if $\prod_{i} \varepsilon_{i}=\mathbb{1}$ the estimates have been product-normalized, anchoring the $\varepsilon_{i}$ and resolving the identification problem. 
To obtain $\gamma_{4}$, the cell entries for the right-most column $(r-1=4)$ in Table 2 must be computed. Starting with $\gamma_{5-1}^{(i)}=\frac{\partial \gamma_{5}}{\partial \varepsilon_{i}}=\gamma_{4}^{(i)}, \gamma_{5}^{(i)}=0$

From Equation 26, $\gamma_{4}^{(i)}=\left(\varepsilon_{1} \varepsilon_{2} \varepsilon_{3} \varepsilon_{4} \varepsilon_{5}-0\right) / \varepsilon_{i}$. Then

$\gamma_{4}^{(1)}=\varepsilon_{2} \varepsilon_{3} \varepsilon_{4} \varepsilon_{5}=.2847$
$\gamma_{4}^{(2)}=\varepsilon_{1} \varepsilon_{3} \varepsilon_{4} \varepsilon_{5}=1.6076$
$\gamma_{4}^{(3)}=\varepsilon_{1} \varepsilon_{2} \varepsilon_{4} \varepsilon_{5}=3.4414$
$\gamma_{4}^{(4)}=\varepsilon_{1} \varepsilon_{2} \varepsilon_{3} \varepsilon_{5}=1.1831$
$\gamma_{4}^{(5)}=\varepsilon_{1} \varepsilon_{2} \varepsilon_{3} \varepsilon_{4}=.5361$.

Note that the values of $\gamma_{4}^{(i)}$ are the reciprocals of $\varepsilon_{i}$; for example,

$\gamma_{4}^{(1)}=.2847=\frac{1}{3.5118}$.

From Equation 27,

$$
\begin{aligned}
\gamma_{4} & =\left(\varepsilon_{2} \varepsilon_{3} \varepsilon_{4} \varepsilon_{5}+\varepsilon_{1} \varepsilon_{3} \varepsilon_{4} \varepsilon_{5}+\varepsilon_{1} \varepsilon_{2} \varepsilon_{4} \varepsilon_{5}+\varepsilon_{1} \varepsilon_{2} \varepsilon_{3} \varepsilon_{5}+\varepsilon_{1} \varepsilon_{2} \varepsilon_{3} \varepsilon_{4}\right) / 1 \\
& =.2847+1.6076+3.4414+1.1831+.5362=7.0529 .
\end{aligned}
$$

For completeness, $\gamma_{3}$ is recalculated using the high-to-low approach. The values of $\gamma_{3}^{(i)}$ are obtained using Equation 26:

$$
\begin{aligned}
\gamma_{3}^{(1)} & =\left[\gamma_{4}-\gamma_{4}^{(1)}\right] / \varepsilon_{1} \\
& =\left[\varepsilon_{2} \varepsilon_{3} \varepsilon_{4} \varepsilon_{5}+\varepsilon_{1} \varepsilon_{3} \varepsilon_{4} \varepsilon_{5}+\varepsilon_{1} \varepsilon_{2} \varepsilon_{4} \varepsilon_{5}+\varepsilon_{1} \varepsilon_{2} \varepsilon_{3} \varepsilon_{5}+\varepsilon_{1} \varepsilon_{2} \varepsilon_{3} \varepsilon_{4}-\varepsilon_{2} \varepsilon_{3} \varepsilon_{4} \varepsilon_{5}\right] / \varepsilon_{1} \\
& =\varepsilon_{3} \varepsilon_{4} \varepsilon_{5}+\varepsilon_{2} \varepsilon_{4} \varepsilon_{5}+\varepsilon_{2} \varepsilon_{3} \varepsilon_{5}+\varepsilon_{2} \varepsilon_{3} \varepsilon_{4} \\
& =[7.0529-2847] / 3.5118 \\
& =1.9273 \\
\gamma_{3}^{(2)} & =\left[\gamma_{4}-\gamma_{4}^{(2)}\right] / \varepsilon_{2} \\
& =\left[\varepsilon_{2} \varepsilon_{3} \varepsilon_{4} \varepsilon_{5}+\varepsilon_{1} \varepsilon_{3} \varepsilon_{4} \varepsilon_{5}+\varepsilon_{1} \varepsilon_{2} \varepsilon_{4} \varepsilon_{5}+\varepsilon_{1} \varepsilon_{2} \varepsilon_{3} \varepsilon_{5}+\varepsilon_{1} \varepsilon_{2} \varepsilon_{3} \varepsilon_{4}-\varepsilon_{1} \varepsilon_{3} \varepsilon_{4} \varepsilon_{5}\right] / \varepsilon_{2} \\
& =\varepsilon_{3} \varepsilon_{4} \varepsilon_{5}+\varepsilon_{1} \varepsilon_{4} \varepsilon_{5}+\varepsilon_{1} \varepsilon_{3} \varepsilon_{5}+\varepsilon_{1} \varepsilon_{3} \varepsilon_{4} \\
& =[7.0529-1.6076] / .6219 \\
& =8.7560 \\
\gamma_{3}^{(3)} & =\left[\gamma_{4}-\gamma_{4}^{(3)}\right] / \varepsilon_{3} \\
& =\left[\varepsilon_{2} \varepsilon_{3} \varepsilon_{4} \varepsilon_{5}+\varepsilon_{1} \varepsilon_{3} \varepsilon_{4} \varepsilon_{5}+\varepsilon_{1} \varepsilon_{2} \varepsilon_{4} \varepsilon_{5}+\varepsilon_{1} \varepsilon_{2} \varepsilon_{3} \varepsilon_{5}+\varepsilon_{1} \varepsilon_{2} \varepsilon_{3} \varepsilon_{4}-\varepsilon_{1} \varepsilon_{2} \varepsilon_{4} \varepsilon_{5}\right] / \varepsilon_{3} \\
& =\varepsilon_{2} \varepsilon_{4} \varepsilon_{5}+\varepsilon_{1} \varepsilon_{4} \varepsilon_{5}+\varepsilon_{1} \varepsilon_{2} \varepsilon_{5}+\varepsilon_{1} \varepsilon_{2} \varepsilon_{4} \\
& =[7.0529-3.4414] / 2905 \\
& =12.4319 \\
\gamma_{3}^{(4)} & =\left[\gamma_{4}-\gamma_{4}^{(4)}\right] / \varepsilon_{4} \\
& =\left[\varepsilon_{2} \varepsilon_{3} \varepsilon_{4} \varepsilon_{5}+\varepsilon_{1} \varepsilon_{3} \varepsilon_{4} \varepsilon_{5}+\varepsilon_{1} \varepsilon_{2} \varepsilon_{4} \varepsilon_{5}+\varepsilon_{1} \varepsilon_{2} \varepsilon_{3} \varepsilon_{5}+\varepsilon_{1} \varepsilon_{2} \varepsilon_{3} \varepsilon_{4}-\varepsilon_{1} \varepsilon_{2} \varepsilon_{3} \varepsilon_{5}\right] / \varepsilon_{4} \\
& =\varepsilon_{2} \varepsilon_{3} \varepsilon_{5}+\varepsilon_{1} \varepsilon_{3} \varepsilon_{5}+\varepsilon_{1} \varepsilon_{2} \varepsilon_{5}+\varepsilon_{1} \varepsilon_{2} \varepsilon_{3} \\
& =[7.0529-1.1831] / 8450 \\
& =6.9465 \\
\gamma_{3}^{(5)} & =\left[\gamma_{4}-\gamma_{4}^{(5)}\right] / \varepsilon_{5} \\
& =\left[\varepsilon_{2} \varepsilon_{3} \varepsilon_{4} \varepsilon_{5}+\varepsilon_{1} \varepsilon_{3} \varepsilon_{4} \varepsilon_{5}+\varepsilon_{1} \varepsilon_{2} \varepsilon_{4} \varepsilon_{5}+\varepsilon_{1} \varepsilon_{2} \varepsilon_{3} \varepsilon_{5}+\varepsilon_{1} \varepsilon_{2} \varepsilon_{3} \varepsilon_{4}-\varepsilon_{1} \varepsilon_{2} \varepsilon_{3} \varepsilon_{4}\right] / \varepsilon_{5}
\end{aligned}
$$




$$
\begin{aligned}
& =\varepsilon_{2} \varepsilon_{3} \varepsilon_{4}+\varepsilon_{1} \varepsilon_{3} \varepsilon_{4}+\varepsilon_{1} \varepsilon_{2} \varepsilon_{4}+\varepsilon_{1} \varepsilon_{2} \varepsilon_{3} \\
& =[7.0529-.5361] / 1.8648 \\
& =3.4946 .
\end{aligned}
$$

When the $\gamma_{3}^{(i)}$ are summed over $i$, each triplet appears twice. From Equation 27,

$$
(5-3) \gamma_{3}=\sum_{i=1}^{5} \gamma_{3}^{(i)} \text { and } \gamma_{3}=\frac{\sum_{i=1}^{5} \gamma_{3}^{(i)}}{2}=\frac{33.5562}{2}=16.7781
$$

which is the same value obtained using the low-to-high part.

Because the midpoint $\gamma_{r}$ will have the largest value and has been calculated by two different approaches, it can be used to test the accuracy of the computations. The GAMMA computer program uses the following check:

$$
\text { TEST }=\left|1-\frac{\gamma_{3}^{\prime}}{\gamma_{3}}\right|=\left|1-\frac{16.7781}{16.7781}\right|=0
$$

where $\gamma_{3}^{\prime}$ is the estimate obtained from the high-to-low part. If TEST $>.01$, its value is reported to alert the user to possible rounding errors.

\section{Computing EST's Using the Summation Algorithm}

Gustafsson (1977) suggested that the summation algorithm (Fischer, 1974) be used to compute the $\gamma_{r}$ and their derivatives because it is less subject to the effects of rounding error than the difference algorithm because no subtraction is involved. This algorithm is based on the following relationship (Fischer, 1974, p. 250, eq. 14.3.11):

$\gamma_{r}\left(\varepsilon_{1}, \varepsilon_{2}, \ldots, \varepsilon_{m}\right)=\gamma_{r}\left(\varepsilon_{1}, \varepsilon_{2}, \ldots, \varepsilon_{m-1}\right)+\varepsilon_{m} \gamma_{r-1}\left(\varepsilon_{1}, \varepsilon_{2}, \ldots, \varepsilon_{m-1}\right)$,

where $0 \leq r \leq m ; m=1,2, \ldots, k$; and $m \leq r$. The recursion involves computing the term on the left-hand side of Equation 35 in stages. In particular, for each increase in $r$, the two terms on the right-hand side of Equation 35 are computed for each possible value of $m \leq r$. The process stops whenever the term on the left-hand side of Equation 35 contains $m$ terms.

The recursion begins by establishing an ESF of order 1 based on a single item, say $\varepsilon_{1}$. Using the LSAT- 6 data, for $r=m=1, m-1=0, \gamma_{0}\left(\varepsilon_{1}\right)=1$ (from Table 1), $\gamma_{1}\left(\varepsilon_{1}, \ldots, \varepsilon_{m-1}\right)=0$, and from Equation 35, $\gamma_{1}\left(\varepsilon_{1}\right)=$ $0+\left(\varepsilon_{1}\right)(1)=\varepsilon_{1}=3.5118$. Because this expression does not yet contain the desired $m=5$ terms, the process continues. Adding a second item parameter, $\varepsilon_{2}$, means $r$ increments to 2 and Equation 35 is used for $m=1$ and then separately for $m=2$.

For $r=m=1, m-1=0$,

$$
\begin{aligned}
\gamma_{0}\left(\varepsilon_{1}, \varepsilon_{2}\right) & =1 \\
\gamma_{1}\left(\varepsilon_{1}, \ldots, \varepsilon_{1}\right) & =\gamma_{1}\left(\varepsilon_{1}\right) \\
\gamma_{1}\left(\varepsilon_{1}, \varepsilon_{2}\right) & =\gamma_{1}\left(\varepsilon_{1}\right)+\varepsilon_{2} \gamma_{0}\left(\varepsilon_{1}\right)=\varepsilon_{1}+\left(\varepsilon_{2}\right)(1)=3.5118+.6219=4.1337 .
\end{aligned}
$$

Given two items, the maximum possible order of the ESF is $r=2$; thus, for $r=m=2, m-1=1$,

$$
\gamma_{2}\left(\varepsilon_{1}, \varepsilon_{2}\right)=\gamma_{2}\left(\varepsilon_{1}\right)+\varepsilon_{2} \gamma_{1}\left(\varepsilon_{1}\right)
$$

but $\gamma_{2}\left(\varepsilon_{1}\right)=0$; thus, 
$\gamma_{2}\left(\varepsilon_{1}, \varepsilon_{2}\right)=0+\varepsilon_{2} \varepsilon_{1}=\varepsilon_{1} \varepsilon_{2}=(3.5118)(.6219)=2.1840$

Adding a third parameter, $\varepsilon_{3}$, means $r$ can be 1,2 , or 3 ; thus, for $r=m=1, m-1=0$,

$$
\begin{aligned}
\gamma_{0}\left(\varepsilon_{1}, \varepsilon_{2}, \varepsilon_{3}\right) & =1 \\
\gamma_{1}\left(\varepsilon_{1}, \varepsilon_{2}, \varepsilon_{3}\right) & =\gamma_{1}\left(\varepsilon_{1}, \varepsilon_{2}\right)+\varepsilon_{3} \gamma_{0}\left(\varepsilon_{1}, \varepsilon_{2}\right) \\
& =\varepsilon_{1}+\varepsilon_{2}+\left(\varepsilon_{3}\right)(1)=\varepsilon_{1}+\varepsilon_{2}+\varepsilon_{3} \\
& =4.1337+.2905=4.4242 .
\end{aligned}
$$

For $r=m=2, m-1=1$,

$$
\begin{aligned}
\gamma_{2}\left(\varepsilon_{1}, \varepsilon_{2}, \varepsilon_{3}\right) & =\gamma_{2}\left(\varepsilon_{1}, \varepsilon_{2}\right)+\varepsilon_{3} \gamma_{1}\left(\varepsilon_{1}, \varepsilon_{2}\right) \\
& =\varepsilon_{1} \varepsilon_{2}+\varepsilon_{3}\left(\varepsilon_{1}+\varepsilon_{2}\right)=\varepsilon_{1} \varepsilon_{2}+\varepsilon_{1} \varepsilon_{3}+\varepsilon_{2} \varepsilon_{3} \\
& =2.1840+.2905(4.1337) \\
& =2.1840+1.2008=3.3848
\end{aligned}
$$

For $r=m=3, m-1=2$,

$\gamma_{3}\left(\varepsilon_{1}, \varepsilon_{2}, \varepsilon_{3}\right)=\gamma_{3}\left(\varepsilon_{1}, \varepsilon_{2}\right)+\varepsilon_{3} \gamma_{2}\left(\varepsilon_{1}, \varepsilon_{2}\right)$

but $\gamma_{3}\left(\varepsilon_{1}, \varepsilon_{2}\right)=0$, then

$\gamma_{3}\left(\varepsilon_{1}, \varepsilon_{2}, \varepsilon_{3}\right)=\varepsilon_{1} \varepsilon_{2} \varepsilon_{3}=.2905(2.1840)=.6345$.

Adding a fourth parameter $\varepsilon_{4}$ yields, for $r=m=1, m-1=0$,

$$
\begin{aligned}
\gamma_{0}\left(\varepsilon_{1}, \varepsilon_{2}, \varepsilon_{3}, \varepsilon_{4}\right) & =\mathbb{1} \\
\gamma_{1}\left(\varepsilon_{1}, \varepsilon_{2}, \varepsilon_{3}, \varepsilon_{4}\right) & =\gamma_{1}\left(\varepsilon_{1}, \varepsilon_{2}, \varepsilon_{3}\right)+\varepsilon_{4} \gamma_{0}\left(\varepsilon_{1}, \varepsilon_{2}, \varepsilon_{3}\right)=\varepsilon_{1}+\varepsilon_{2}+\varepsilon_{3}+\varepsilon_{4} \\
& =4.4242+.8450(1)=5.2692 .
\end{aligned}
$$

For $r=m=2, m-1=1$,

$$
\begin{aligned}
\gamma_{2}\left(\varepsilon_{1}, \varepsilon_{2}, \varepsilon_{3}, \varepsilon_{4}\right) & =\gamma_{2}\left(\varepsilon_{1}, \varepsilon_{2}, \varepsilon_{3}\right)+\varepsilon_{4} \gamma_{1}\left(\varepsilon_{1}, \varepsilon_{2}, \varepsilon_{3}\right) \\
& =\varepsilon_{1} \varepsilon_{2}+\varepsilon_{1} \varepsilon_{3}+\varepsilon_{2} \varepsilon_{3}+\varepsilon_{4} \varepsilon_{1}+\varepsilon_{4} \varepsilon_{2}+\varepsilon_{4} \varepsilon_{3} \\
& =3.3848+84.50(4.4242) \\
& =3.3848+3.7385=7.1233 .
\end{aligned}
$$

For $r=m=3, m-1=2$,

$$
\begin{aligned}
\gamma_{3}\left(\varepsilon_{1}, \varepsilon_{2}, \varepsilon_{3}, \varepsilon_{4}\right) & =\gamma_{3}\left(\varepsilon_{1}, \varepsilon_{2}, \varepsilon_{3}\right)+\varepsilon_{4} \gamma_{2}\left(\varepsilon_{1}, \varepsilon_{2}, \varepsilon_{3}\right) \\
& =\varepsilon_{1} \varepsilon_{2} \varepsilon_{3}+\varepsilon_{4} \varepsilon_{1} \varepsilon_{2}+\varepsilon_{4} \varepsilon_{1} \varepsilon_{3}+\varepsilon_{4} \varepsilon_{2} \varepsilon_{3} \\
& =.63445+.8450(3.3848) \\
& =.6345+2.8602=3.4946 .
\end{aligned}
$$

For $r=m=4, m-1=3$,

$$
\begin{aligned}
\gamma_{4}\left(\varepsilon_{1}, \varepsilon_{2}, \varepsilon_{3}, \varepsilon_{4}\right) & =\gamma_{4}\left(\varepsilon_{1}, \varepsilon_{2}, \varepsilon_{3}\right)+\varepsilon_{4} \gamma_{3}\left(\varepsilon_{1}, \varepsilon_{2}, \varepsilon_{3}\right) \text { but } \gamma_{4}\left(\varepsilon_{1}, \varepsilon_{2}, \varepsilon_{3}\right)=0 \\
& =\varepsilon_{1} \varepsilon_{2} \varepsilon_{3} \varepsilon_{4} \\
& =.8450(.6345)=.5361 .
\end{aligned}
$$

Adding a fifth parameter $\varepsilon_{5}$ yields, for $r=m=1, m-1=0$,

$$
\begin{aligned}
\gamma_{0}\left(\varepsilon_{1}, \varepsilon_{2}, \varepsilon_{3}, \varepsilon_{4}, \varepsilon_{5}\right) & =1 \\
\gamma_{1}\left(\varepsilon_{1}, \varepsilon_{2}, \varepsilon_{3}, \varepsilon_{4}, \varepsilon_{5}\right) & =\gamma_{1}\left(\varepsilon_{1}, \varepsilon_{2}, \varepsilon_{3}, \varepsilon_{4}\right)+\varepsilon_{5} \gamma_{0}\left(\varepsilon_{1}, \varepsilon_{2}, \varepsilon_{3}, \varepsilon_{4}\right) \\
& =\varepsilon_{1}+\varepsilon_{2}+\varepsilon_{3}+\varepsilon_{4}+\varepsilon_{5} \\
& =5.2692+1.8648=7.1340
\end{aligned}
$$


which has $\left(\begin{array}{l}5 \\ 1\end{array}\right)$ terms.

For $r=m=2, m-1=1$,

$$
\begin{aligned}
\gamma_{2}\left(\varepsilon_{1}, \varepsilon_{2}, \varepsilon_{3}, \varepsilon_{4}, \varepsilon_{5}\right) & =\gamma_{2}\left(\varepsilon_{1}, \varepsilon_{2}, \varepsilon_{3}, \varepsilon_{4}\right)+\varepsilon_{5} \gamma_{1}\left(\varepsilon_{1}, \varepsilon_{2}, \varepsilon_{3}, \varepsilon_{4}\right) \\
& =\varepsilon_{1} \varepsilon_{2}+\varepsilon_{1} \varepsilon_{3}+\varepsilon_{1} \varepsilon_{4}+\varepsilon_{2} \varepsilon_{3}+\varepsilon_{2} \varepsilon_{4}+\varepsilon_{3} \varepsilon_{4}+\varepsilon_{5} \varepsilon_{1}+\varepsilon_{5} \varepsilon_{2}+\varepsilon_{5} \varepsilon_{3}+\varepsilon_{5} \varepsilon_{4} \\
& =7.1233+1.8648(5.2692)=16.9493
\end{aligned}
$$

which has $\left(\begin{array}{l}5 \\ 2\end{array}\right)$ terms.

The remaining $\gamma_{r}$ are obtained using Equation 35 and the terms computed above.

For $r=m=3, m-1=2$,

$$
\begin{aligned}
\gamma_{3}\left(\varepsilon_{1}, \varepsilon_{2}, \varepsilon_{3}, \varepsilon_{4}, \varepsilon_{5}\right)= & \gamma_{3}\left(\varepsilon_{1}, \varepsilon_{2}, \varepsilon_{3}, \varepsilon_{4}\right)+\varepsilon_{5} \gamma_{2}\left(\varepsilon_{10} \varepsilon_{2}, \varepsilon_{3}, \varepsilon_{4}\right) \\
= & \varepsilon_{1} \varepsilon_{2} \varepsilon_{3}+\varepsilon_{1} \varepsilon_{2} \varepsilon_{4}+\varepsilon_{1} \varepsilon_{3} \varepsilon_{4}+\varepsilon_{2} \varepsilon_{3} \varepsilon_{4}+\varepsilon_{5} \varepsilon_{1} \varepsilon_{2}+\varepsilon_{5} \varepsilon_{1} \varepsilon_{3}+\varepsilon_{5} \varepsilon_{2} \varepsilon_{3} \\
& +\varepsilon_{5} \varepsilon_{4} \varepsilon_{1}+\varepsilon_{5} \varepsilon_{4} \varepsilon_{2}+\varepsilon_{5} \varepsilon_{4} \varepsilon_{3} \\
= & 3.4946+1.8648(7.1233)=16.7781,
\end{aligned}
$$

which has $\left(\begin{array}{l}5 \\ 3\end{array}\right)$ terms.

$$
\text { For } r=m=4, m-1=3 \text {, }
$$

$$
\begin{aligned}
\gamma_{4}\left(\varepsilon_{1}, \varepsilon_{2}, \varepsilon_{3}, \varepsilon_{4}, \varepsilon_{5}\right) & =\gamma_{4}\left(\varepsilon_{1}, \varepsilon_{2}, \varepsilon_{3}, \varepsilon_{4}\right)+\varepsilon_{5} \gamma_{3}\left(\varepsilon_{1}, \varepsilon_{2}, \varepsilon_{3}, \varepsilon_{4}\right) \\
& =\varepsilon_{1} \varepsilon_{2} \varepsilon_{3} \varepsilon_{4}+\varepsilon_{5} \varepsilon_{1} \varepsilon_{2} \varepsilon_{3}+\varepsilon_{5} \varepsilon_{1} \varepsilon_{2} \varepsilon_{4}+\varepsilon_{5} \varepsilon_{1} \varepsilon_{3} \varepsilon_{4}+\varepsilon_{5} \varepsilon_{2} \varepsilon_{3} \varepsilon_{4} \\
& =.5361+1.8648(3.4946)=7.0529
\end{aligned}
$$

which has $\left(\begin{array}{l}5 \\ 4\end{array}\right)$ terms.

For $r=m=5, m-1=4$,

$$
\gamma_{5}\left(\varepsilon_{1}, \varepsilon_{2}, \varepsilon_{3}, \varepsilon_{4}, \varepsilon_{5}\right)=\gamma_{5}\left(\varepsilon_{1}, \varepsilon_{2}, \varepsilon_{3}, \varepsilon_{4}\right)+\varepsilon_{5} \gamma_{4}\left(\varepsilon_{1}, \varepsilon_{2}, \varepsilon_{3}, \varepsilon_{4}\right)
$$

but $\gamma_{5}\left(\varepsilon_{1}, \varepsilon_{2}, \varepsilon_{3}, \varepsilon_{4}\right)=0$, then

$$
\begin{aligned}
\gamma_{5}\left(\varepsilon_{1}, \varepsilon_{2}, \varepsilon_{3}, \varepsilon_{4}, \varepsilon_{5}\right) & =0+\varepsilon_{5} \varepsilon_{1} \varepsilon_{2} \varepsilon_{3} \varepsilon_{4}=\varepsilon_{1} \varepsilon_{2} \varepsilon_{3} \varepsilon_{4} \varepsilon_{5} \\
& =0+1.8648(.5361)=.9997,
\end{aligned}
$$

which has $\left(\begin{array}{l}5 \\ 5\end{array}\right)$ terms.

Having included all 5 items $(m=5)$, the recursion process is terminated and the computed values of the $\gamma_{r}$ are those reported in Table 3. Although the implementation of the summation algorithm in the GAM subroutine (Fischer, 1974, p. 544) required only 24 lines of FORTRAN code, it is computationally more demanding than the difference algorithm because it computes the $\gamma_{r}$ for $r=1,2, \ldots, m$ as $m$ varies from 1 to $k$.

\section{A Network Representation of the Summation Algorithm}

Liou \& Chang (1992) developed a directed acyclic network representation of the possible item response vectors for $k$ dichotomously scored items. Such a network consists of $n+1$ stages beginning at stage 0 with a single node. Each node at stage $i$ generates two successor nodes at stage $i+1$. Two directed arcs connect the node at a lower indexed stage to its two successors. Liou (personal communication, May, 1996) suggested that this network approach also could be used to represent the computation of the ESFs using the summation algorithm.

The network for a 5 -item test is shown in Figure 1. A node in the network is denoted by $\left(m, r_{m}\right)$ where the first letter denotes the stage of the network and the second indicates the order of the ESF at that node. For example, node $(4,2)$ indicates that there are four items involved in the ESF at that node and an ESF of order two corresponds to the node [i.e., $\left.\gamma_{2}\left(\varepsilon_{1}, \varepsilon_{2}, \varepsilon_{3}, \varepsilon_{4}\right)\right]$. The line connecting two successive nodes is an arc whose length is defined by $\varepsilon_{m+1}^{u_{m+1}}$ where $u_{m+1}=r_{m+1}-r_{m}$.

If the node at the end of the arc is below and to the left of the current node, then $u_{m+1}=0$. For example, 
Fing

Directed Acyclic Network Representation of the Computation of ESFs for 5 lrems

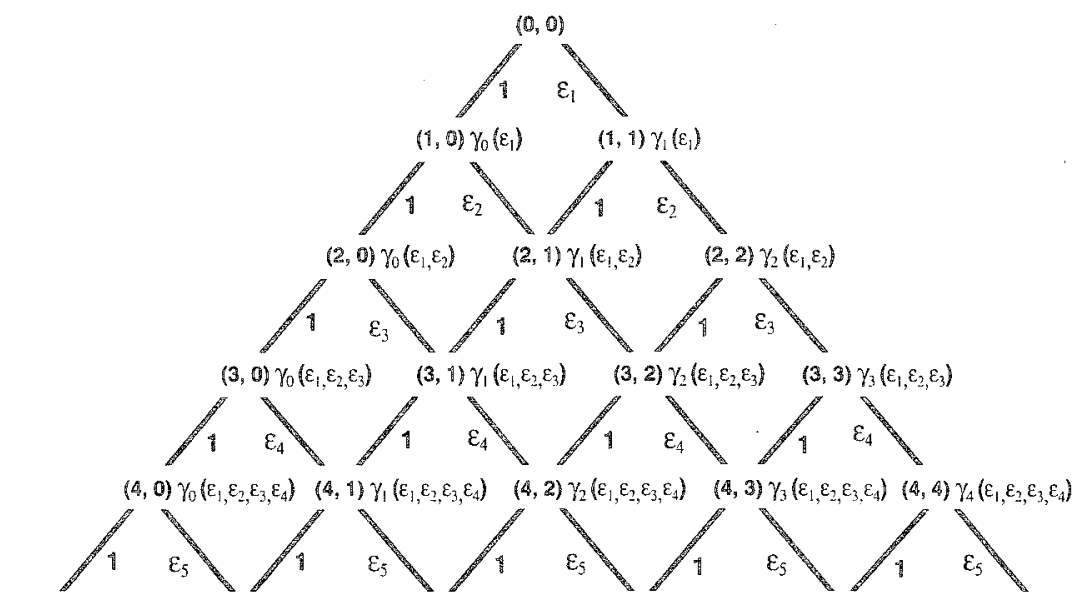

$(5,0) \gamma_{0}\left(\varepsilon_{1}, \varepsilon_{2}, \varepsilon_{3}, \varepsilon_{4}, \varepsilon_{5}\right)(5,1) \gamma_{1}\left(\varepsilon_{1}, \varepsilon_{2}, \varepsilon_{3}, \varepsilon_{4}, \varepsilon_{5}\right)(5,2) \gamma_{2}\left(\varepsilon_{1}, \varepsilon_{2}, \varepsilon_{3}, \varepsilon_{4}, \varepsilon_{5}\right)(5,3) \gamma_{3}\left(\varepsilon_{1}, \varepsilon_{2}, \varepsilon_{3}, \varepsilon_{4}, \varepsilon_{5}\right)(5,4) \gamma_{4}\left(\varepsilon_{1}, \varepsilon_{2}, \varepsilon_{3}, \varepsilon_{4}, \varepsilon_{5}\right)(5,5) \gamma_{5}\left(\varepsilon_{1}, \varepsilon_{2}, \varepsilon_{3}, \varepsilon_{4}, \varepsilon_{5}\right)$

the length of the are connecting nodes $(2,1)$ and $(3,1)$ is $\varepsilon_{3}^{1-1}=\varepsilon_{3}^{0}=1$. If the node at the end of the arc is below and to the right, say $(2,1)$ to $(3,2)$, then the length is $\varepsilon_{3}^{2-1}=\varepsilon_{3}^{1}=\varepsilon_{3}$. The length of a path from the initial node $(0,0)$ to a node in a higher indexed stage is the product of the lengths of the arcs comprising the path. For example, the length of the path $(0,0) \rightarrow(1,0) \rightarrow(2,0) \rightarrow(3,1)=1 \times 1 \times \varepsilon_{3}=\varepsilon_{3}$. The length of the path from $(0,0) \rightarrow(1,1) \rightarrow(2,1)=\varepsilon_{1} \times 1=\varepsilon_{1}$. The ESF at each node is defined as the sum of the lengths of all the paths connecting the initial node $(0,0)$ to that node. For example, at node $(2,1)$,

$\gamma_{1}\left(\varepsilon_{1}, \varepsilon_{2}\right)=\left(1 \cdot \varepsilon_{2}\right)+\left(\varepsilon_{1} \cdot 1\right)=\varepsilon_{1}+\varepsilon_{2}$,

and at node $(3,1)$,

$\gamma_{1}\left(\varepsilon_{1}, \varepsilon_{2}, \varepsilon_{3}\right)=\left(1 \cdot 1 \cdot \varepsilon_{3}\right)+\left(1 \cdot \varepsilon_{2} \cdot 1\right)+\left(\varepsilon_{1} \cdot 1 \cdot 1\right)=\varepsilon_{1}+\varepsilon_{2}+\varepsilon_{3}$.

Although the ESF at any node can be found in this manner, it is simpler to define the ESE at a node in terms of the ESFS of the two nodes in the preceding stage that are connected to it. Thus, at node $\left(m, r_{m}\right)$ the two nodes connected to it are $\left(m-1, r_{m-1}\right)$ and $\left(m-1, r_{m}\right)$ and the ESF is given by

$\gamma_{r}\left(\varepsilon_{1}, \varepsilon_{2}, \ldots, \varepsilon_{m-1}\right) \varepsilon_{m}+\gamma_{r}\left(\varepsilon_{1}, \varepsilon_{2}, \ldots, \varepsilon_{m-1}\right)=\gamma_{r}\left(\varepsilon_{1}, \varepsilon_{2}, \ldots, \varepsilon_{m}\right)$,

where the first term corresponds to the path coming from the upper left and the second term to that coming from the upper right. Note that Equation 55 is identical to Equation 35, which defines the summation algorithm. For nodes on the outer edges of the network, the term in Equation 55 corresponding to the nonexistent arc is set to 0. For example at $(3,3)$ the ESF is $\gamma_{2}\left(\varepsilon_{1}, \varepsilon_{2}\right) \times \varepsilon_{3}+0$. At $(3,0)$ the ESF is $0+\gamma_{0}\left(\varepsilon_{1}, \varepsilon_{2}\right)$.

Implementing the summation algorithm using the network approach is straightforward. The stages advance from node $(0,0)$ down the left-hand edge of the network depicted in Figure 1. Increasing the stage is the same as including the next item within the summation algorithm. At each stage, the network is "swept" horizontally from left to right and each of the $\gamma_{r}$ possible at that stage are computed using the upper-leftupperright paradigm described above. This process is repeated stage by stage until all $k$ items have been included. Thus, the $\gamma_{r}$ computed across the bottom of the network in the last stage are the desired ESFs. It is important to recognize that this process constructs the network of Figure 1 from the top of the diagram to the bottom of the diagram. Liou (personal communication, May, 1996) provided a FORTRAN computer program that imple- 
ments this directed acyclic network approach to computing the ESFs in only 15 lines of code.

\section{An Allernative Summation Algorithm}

Verhelst et al. (1984) provided a reformulation of the summation algorithm in which the upper triangle of a $k \times k$ matrix is computed with elements

$$
t_{i j}= \begin{cases}\sum_{s=1}^{j} \varepsilon_{s}=t_{i, j-1}+\varepsilon_{j} & (i=1) \\ t_{i, j-1}+\varepsilon_{j} t_{(i-1, j-1)} & (1<i<j), \\ \frac{j}{\prod_{s}} \varepsilon_{s}=t_{j-1, j-1} \varepsilon_{j} & (i=j)\end{cases}
$$

where $i=1,2, \ldots, k$ denotes a row, and $j=1,2, \ldots, k$ denotes a column. When a subscript identifies a nonexistent cell, $t_{i j}=0$. The $\hat{t}_{i j}$ show that a recursive process is being defined. In the row indexed by $i=1$, the sum of the $\varepsilon$ parameters is created item by item for $j=1,2, \ldots, k$. For $1<i<j$, the definition of $t_{i j}$ is the same as $\gamma_{r}\left(\varepsilon_{1}, \varepsilon_{2}, \ldots, \varepsilon_{m}\right)$ in Equation 35 for the summation algorithm, and the values computed in the preceding row are used. When $i=j, \mathbb{t}_{i j}$ is simply the product of the set of $\varepsilon_{i}$ involved in the row-column combination. Thus, proceeding across columns within a row, and then down by rows produces the $\gamma_{r}$ in the right-most column of the matrix.

To implement this algorithm, the item parameters are initially placed in the order $\varepsilon_{1}, \varepsilon_{2}, \varepsilon_{3}, \varepsilon_{4}, \varepsilon_{5}$. For the first row of the matrix,

$$
\begin{aligned}
& i=1, j=1, \quad t_{1,1}=\sum_{s=1}^{1} \varepsilon_{s}=t_{1,0}+\varepsilon_{1}=0+\varepsilon_{1}=\varepsilon_{1} \\
& i=1, j=2, \quad t_{1,2}=\sum_{s=1}^{2} \varepsilon_{s}=t_{1,1}+\varepsilon_{2}=\varepsilon_{1}+\varepsilon_{2} \\
& i=1, j=3, \quad t_{1,3}=\sum_{s=1}^{3} \varepsilon_{s}=t_{1,2}+\varepsilon_{3}=\varepsilon_{1}+\varepsilon_{2}+\varepsilon_{3} \\
& i=1, j=4, \quad t_{1,4}=\sum_{s=1}^{4} \varepsilon_{s}=t_{1,3}+\varepsilon_{4}=\varepsilon_{1}+\varepsilon_{2}+\varepsilon_{3}+\varepsilon_{4} \\
& i=1, j=5, \quad t_{1,5}=\sum_{s=1}^{5} \varepsilon_{s}=t_{1,4}+\varepsilon_{5}=\varepsilon_{1}+\varepsilon_{2}+\varepsilon_{3}+\varepsilon_{4}+\varepsilon_{5} .
\end{aligned}
$$

For the second row,

$$
\begin{array}{ll}
i=2, j=2, & t_{2,2}=\varepsilon_{2} t_{1,1}=\varepsilon_{1} \varepsilon_{2} \\
i=2, j=3, & t_{2,3}=t_{2,2}+\varepsilon_{3} t_{1,2}=t_{2,2}+\varepsilon_{3}\left(\varepsilon_{1}+\varepsilon_{2}\right)=t_{2,2}+\varepsilon_{1} \varepsilon_{3}+\varepsilon_{2} \varepsilon_{3} \\
i=2, j=4, & t_{2,4}=t_{2,3}+\varepsilon_{4} t_{1,3}=t_{2,3}+\varepsilon_{1} \varepsilon_{4}+\varepsilon_{2} \varepsilon_{4}+\varepsilon_{3} \varepsilon_{4} \\
i=2, j=5, & t_{2,5}=t_{2,4}+\varepsilon_{5} t_{1,4}=t_{2,4}+\varepsilon_{1} \varepsilon_{5}+\varepsilon_{2} \varepsilon_{5}+\varepsilon_{3} \varepsilon_{5}+\varepsilon_{4} \varepsilon_{5} .
\end{array}
$$

For the third row,

$$
\begin{array}{ll}
i=3, j=3, & t_{3,3}=t_{2,2} \varepsilon_{3}=\varepsilon_{1} \varepsilon_{2} \varepsilon_{3} \\
i=3, j=4, & t_{3,4}=t_{3,3}+\varepsilon_{4} t_{2,3}=t_{3,3}+\varepsilon_{1} \varepsilon_{2} \varepsilon_{4}+\varepsilon_{1} \varepsilon_{3} \varepsilon_{4}+\varepsilon_{2} \varepsilon_{3} \varepsilon_{4} \\
i=3, j=5, & t_{3,5}=t_{3,4}+\varepsilon_{5} t_{2,4}=t_{3,4}+\varepsilon_{1} \varepsilon_{2} \varepsilon_{5}+\varepsilon_{1} \varepsilon_{3} \varepsilon_{5}+\varepsilon_{1} \varepsilon_{4} \varepsilon_{5}+\varepsilon_{2} \varepsilon_{3} \varepsilon_{5}+\varepsilon_{2} \varepsilon_{4} \varepsilon_{5}+\varepsilon_{3} \varepsilon_{4} \varepsilon_{5} .
\end{array}
$$


For the fourth row,

$i=4, j=4, \quad t_{4,4}=t_{4,3}+\varepsilon_{4} t_{3,3}=0+\varepsilon_{1} \varepsilon_{2} \varepsilon_{3} \varepsilon_{4}$

$i=4, j=5, \quad t_{4,5}=t_{4,4}+\varepsilon_{5} t_{3,4}=t_{4,4}+\varepsilon_{1} \varepsilon_{2} \varepsilon_{3} \varepsilon_{5}+\varepsilon_{1} \varepsilon_{2} \varepsilon_{4} \varepsilon_{5}+\varepsilon_{1} \varepsilon_{3} \varepsilon_{4} \varepsilon_{5}+\varepsilon_{2} \varepsilon_{3} \varepsilon_{4} \varepsilon_{5}$.

For the fifth row,

$i=5, j=5, \quad t_{5,5}=t_{4,4} \varepsilon_{5}=\varepsilon_{1} \varepsilon_{2} \varepsilon_{3} \varepsilon_{4} \varepsilon_{5}$.

These results are presented in terms of the ESFs and their derivatives in Table 4. Table 4 shows that (1) the $k$ th column (column 5) contains the $\gamma_{r}$, with the order of the ESF corresponding to the index of the row in which it appeared; (2) the $k$ - lith column contains the derivatives with respect to the $\varepsilon_{k}$ of the ESF having the next higher order than the index of the row; (3) the $k-2$ th column contains the second derivatives with respect to $\varepsilon_{k}$ and $\varepsilon_{k-1}$ of the ESF of order 2 higher than the index of the row; (4) the $k$-3th column contains the third derivatives with respect to $\varepsilon_{k}, \varepsilon_{k-1}$, and $\varepsilon_{k-2}$ of the ESF of order 3 higher than the index of the row; and (5) the $k-4$ th column (the first column in Table 4 ) contains a single term, which is the derivative of the ESF of order 4 higher than the index of the row, with respect to $\varepsilon_{k-3}, \varepsilon_{k-2}, \varepsilon_{k-1}$, and $\varepsilon_{k}$.

Table 4

ESFs and Derivatives Yielded by the Verhelst, Glas, \& van der Sluis (1984) Technique for the Item Order $i=1,2,3,4,5$

\begin{tabular}{lccccc}
\hline \hline ESF & \multicolumn{5}{c}{ Item Order } \\
\cline { 2 - 6 } Order & 1 & 2 & 3 & 4 & 5 \\
\hline 1 & $\gamma_{5-4}^{(2,3,4,5)}$ & $\gamma_{4-3}^{(3,4,5)}$ & $\gamma_{3-2}^{(4,5)}$ & $\gamma_{2-1}^{(5)}$ & $\gamma_{1}$ \\
2 & & $\gamma_{5-3}^{(3,4,5)}$ & $\gamma_{4-2}^{(4,5)}$ & $\gamma_{3-1}^{(5)}$ & $\gamma_{2}$ \\
3 & & & $\gamma_{5-2}^{(4,5)}$ & $\gamma_{4-1}^{(5)}$ & $\gamma_{3}$ \\
4 & & & $\gamma_{5-1}^{(5)}$ & $\gamma_{4}$ \\
5 & & & & $\gamma_{5}$ \\
\hline
\end{tabular}

Note that the first derivatives in Table 4 are with respect to only the $k$ th item. To obtain the first derivatives for the remaining $k-1$ items, the process would be repeated using each of the remaining $k-1$ items as the $k$ th item in the list of items; that is, the item order would be cyclically rotated to the right once for each matrix. However, it would not be necessary to compute the $k$ th column, but four more matrices of dimension $k-1 \times k-1$ would have to be computed. Computing the full set of $k$ matrices would yield the ESFS and all of the first derivatives of the $\gamma_{r}$, but the full set of second-and higher-order derivatives would not be produced.

As was the case with Fischer's summation algorithm, this alternative algorithm can be implemented using a network approach. Starting at node $(0,0)$ whose value is always 1 , the network in Figure 1 is "swept" along the path that goes left and down until the last stage is reached at node $(5,0)$. At each node encountered on this path, the corresponding $\gamma_{r}$ is computed using the upper-left/upper-right paradigm described above.

For example, the left and down path originating at $(0,0)$ contains the $\gamma_{0}$ for all $k$ stages. The stages themselves advance down the right-hand edge of the network depicted in Figure 1. Again, increasing the stage is the same as including the next item. Thus, going from node $(0,0)$ to $(1,1)$ includes $\varepsilon_{1}$ and is followed by a "sweep" to the left and down along the path to node $(5,1)$, yielding the $k-(m-1) \gamma_{1}$. This process is repeated stage by stage until all $k$ items have been included. Note that computing the $\gamma_{r}$ along the left and down path is identical to computing the terms in a row of the matrix depicted in Table 4. The ESF 
computed at the end of a path is the $\gamma_{r}$ in the kth column of Table 4 . The $\gamma_{r}$ computed at the nodes along the right edge of the network correspond to the diagonal terms of the matrix in Table 4. It is important to recognize that under the alternative summation algorithm, the network of Figure 1 is constructed using top to bottom paths from left to right across the network. This is in contrast to Fischer's summation algorithm in which the retwork is constructed by rows from top to bottom.

Although using different approaches, both the summation and alternative summation algorithms construct the same network. That the two summation algorithms have been shown to be variations on constructing a network is very important for several reasons. First, it shows that there was a common underlying framework for these two algorithms. Second, it leads to very compact FORTRAN computer programs for evaluating the ESFs. Third, it suggests that other algorithms for evaluating ESFS might be represented by networks and perhaps a common framework could be developed for a variety of such algorithms.

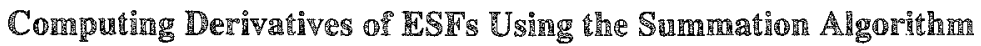

The summation algorithm can also be used to evaluate the first and second derivatives of the ESFs with respect to the item parameters. A very clever approach was used by Fischer (1974, p. 544) in the GAM subroutine to obtain the values of the derivatives. To obtain the first derivatives, simply set the value of the $\varepsilon_{i}$ with which the derivative is to be taken to 0 and execute the summation algorithm for the full set of $k$ items, yielding $\gamma_{r}^{(i)}$ for $r=1,2, \ldots, k$. To obtain the first derivatives with respect to all $k$ item parameters requires repeating this process $k$ times. The second derivatives are obtained by setting the values of a pair of the $\varepsilon_{\text {, say }} \varepsilon_{i}$ and $\varepsilon_{h}$, to 0 and executing the algorithm, which will then yield $\gamma_{r}^{(i, h)}$ for that pair. This process is repeated $k(k-1) / 2$ times to obtain the full set of second derivatives.

To find the first derivative of the ESFs for $r=1,2, \ldots, k$ with respect to $\varepsilon_{1}\left[\right.$ i.e., the $\left.\gamma_{r}^{(1)}\right], \varepsilon_{1}$ is set to 0 and the summation algorithm is initiated using $\varepsilon_{1}$ as the first item to be included. Then, for $r=m=1, m-1=0$,

$$
\begin{aligned}
& \gamma_{0}\left(\varepsilon_{1}\right)=1 \\
& \gamma_{1}\left(\varepsilon_{1}\right)=0 . \\
& \text { Adding } \varepsilon_{2}, r=m=1, m-1=0, \\
& \gamma_{0}\left(\varepsilon_{1}, \varepsilon_{2}\right)=1 \\
& \gamma_{1}\left(\varepsilon_{1}, \varepsilon_{2}\right)=0+.6219(1)+.6219 . \\
& r=m=2, m-1=1, \\
& \gamma_{2}\left(\varepsilon_{1}, \varepsilon_{2}\right)=0+(.6219) 0=0 . \\
& \text { Adding } \varepsilon_{3}, r=m=1, m-1=0, \\
& \gamma_{0}\left(\varepsilon_{1}, \varepsilon_{2}, \varepsilon_{3}\right)=1 \\
& \gamma_{1}\left(\varepsilon_{1}, \varepsilon_{2}, \varepsilon_{3}\right)=.6219+.2905(1)=.9124 . \\
& r=m=2, m-1=1 \\
& \gamma_{2}\left(\varepsilon_{1}, \varepsilon_{2}, \varepsilon_{3}\right)=0+.2905(.6219)=.1807 . \\
& r=m=3, m-1=2, \\
& \gamma_{3}\left(\varepsilon_{1}, \varepsilon_{2}, \varepsilon_{3}\right)=0 . \\
& \text { Adding } \varepsilon_{4}, r=m=1, m-1=0, \\
& \gamma_{0}\left(\varepsilon_{1}, \varepsilon_{2}, \varepsilon_{3}, \varepsilon_{4}\right)=1 \\
& \gamma_{1}\left(\varepsilon_{1}, \varepsilon_{2}, \varepsilon_{3}, \varepsilon_{4}\right)=.9124+.8450(1)=1.7574 . \\
& r=m=2, m-1=1
\end{aligned}
$$


$\gamma_{2}\left(\varepsilon_{1}, \varepsilon_{2}, \varepsilon_{3}, \varepsilon_{4}\right)=.1807+.8450(.9124)=.9516$

$r=m=3, m-1=2$,

$\gamma_{3}\left(\varepsilon_{1}, \varepsilon_{2}, \varepsilon_{3}, \varepsilon_{4}\right)=0+.8450(.1807)=.1527$.

$r=m=4, m-1=3$,

$\gamma_{4}\left(\varepsilon_{1}, \varepsilon_{2}, \varepsilon_{3}, \varepsilon_{4}\right)=0$.

Adding $\varepsilon_{5}, r=m=1, m-1=0$,

$\gamma_{0}\left(\varepsilon_{1}, \varepsilon_{2}, \varepsilon_{3}, \varepsilon_{4}, \varepsilon_{5}\right)=1$

$\gamma_{1}\left(\varepsilon_{1}, \varepsilon_{2}, \varepsilon_{3}, \varepsilon_{4}, \varepsilon_{5}\right)=1.7574+1.8648(1)=3.6222=\frac{\partial \gamma_{2}\left(\varepsilon_{1}, \varepsilon_{2}, \varepsilon_{3}, \varepsilon_{4}, \varepsilon_{5}\right)}{\partial \varepsilon_{1}}=\gamma_{1}^{(1)}$.

$r=m=2, m-1=1$,

$\gamma_{2}\left(\varepsilon_{1}, \varepsilon_{2}, \varepsilon_{3}, \varepsilon_{4}, \varepsilon_{5}\right)=.95164+1.8648(1.7574)=4.2288=\frac{\partial \gamma_{3}\left(\varepsilon_{1}, \varepsilon_{2}, \varepsilon_{3}, \varepsilon_{4}, \varepsilon_{5}\right)}{\partial \varepsilon_{1}}=\gamma_{2}^{(1)}$.

$r=m=3, m-1=2$,

$\gamma_{3}\left(\varepsilon_{1}, \varepsilon_{2}, \varepsilon_{3}, \varepsilon_{4}, \varepsilon_{5}\right)=.1527+1.8648(.9516)=1.9273=\frac{\partial \gamma_{4}\left(\varepsilon_{1}, \varepsilon_{2}, \varepsilon_{3}, \varepsilon_{4}, \varepsilon_{5}\right)}{\partial \varepsilon_{1}}=\gamma_{3}^{(1)}$.

$r=m=4, m-1=3$,

$\gamma_{4}\left(\varepsilon_{1}, \varepsilon_{2}, \varepsilon_{3}, \varepsilon_{4}, \varepsilon_{5}\right)=0+1.8648(.1527)=.2847=\frac{\partial \gamma_{5}\left(\varepsilon_{1}, \varepsilon_{2}, \varepsilon_{3}, \varepsilon_{4}, \varepsilon_{5}\right)}{\partial \varepsilon_{1}}=\gamma_{4}^{(1)}$.

$r=m=5, m-1=4$,

$\gamma_{5}\left(\varepsilon_{1}, \varepsilon_{2}, \varepsilon_{3}, \varepsilon_{4}, \varepsilon_{5}\right)=0=\frac{\partial \gamma_{6}\left(\varepsilon_{1}, \varepsilon_{2}, \varepsilon_{3}, \varepsilon_{4}, \varepsilon_{5}\right)}{\partial \varepsilon_{1}}=\gamma_{5}^{(1)}$.

These values for the first derivatives are the same as those reported for Item 1 in the first row of Table 3. This process would be repeated for each of the remaining items to obtain the first derivatives.

The summation algorithm also can be used to obtain the numerical values of the second derivatives of the $\gamma_{r}$ that are needed when estimating the $\varepsilon_{i}$ using the Newton-Raphson technique. Although the formulas for the second derivatives are known, they are not evaluated using direct substitution of the values of the $\varepsilon_{i}$, Instead, they are computed using the summation algorithm, which is illustrated below using the LSAT- $6 \varepsilon$ parameter estimates.

Taking the second derivatives of $\gamma_{r}$ with respect to $\varepsilon_{i}=\varepsilon_{1}$ and $\varepsilon_{h}=\varepsilon_{2}$, begin by setting the values of these two $\varepsilon$ parameters to 0 and executing the summation algorithm.

Starting with a single item parameter $\varepsilon_{1}$,

$\gamma_{1}\left(\varepsilon_{1}\right)=0, \quad \gamma_{0}\left(\varepsilon_{1}\right)=1, \quad \gamma_{1}\left(\varepsilon_{2}\right)=0, \quad \gamma_{0}\left(\varepsilon_{2}\right)=1, \quad \gamma_{0}\left(\varepsilon_{1}, \varepsilon_{2}\right)=1$.

Including $\varepsilon_{2}$ produces $r=m=1, m-1=0$,

$\gamma_{0}\left(\varepsilon_{1}, \varepsilon_{2}\right)=1$

$\gamma_{1}\left(\varepsilon_{1}, \varepsilon_{2}\right)=\gamma_{1}\left(\varepsilon_{1}\right)+\varepsilon_{2} \gamma_{0}\left(\varepsilon_{1}\right)=0+0(1)=0$

$r=m=2, m-1=1$,

$\gamma_{2}\left(\varepsilon_{1}, \varepsilon_{2}\right)=\gamma_{2}\left(\varepsilon_{1}\right)+\varepsilon_{2} \gamma_{1}\left(\varepsilon_{1}\right)=0$. 
Including $\varepsilon_{3}$ produces $r=m=1, m-1=0$,

$\gamma_{0}\left(\varepsilon_{1}, \varepsilon_{2}, \varepsilon_{3}\right)=1$

$\gamma_{1}\left(\varepsilon_{1}, \varepsilon_{2}, \varepsilon_{3}\right)=\gamma_{1}\left(\varepsilon_{2}\right)+\varepsilon_{3} \gamma_{0}\left(\varepsilon_{1}, \varepsilon_{2}\right)=0+.2905(1)=.2905$.

$r=m=2, m-1=1$,

$\gamma_{2}\left(\varepsilon_{1}, \varepsilon_{2}, \varepsilon_{3}\right)=0+\varepsilon_{3} \gamma_{1}\left(\varepsilon_{1}, \varepsilon_{2}\right)=0$.

$r=m=3, m-1=2$,

$\gamma_{3}\left(\varepsilon_{1}, \varepsilon_{2}, \varepsilon_{3}\right)=0+\varepsilon_{3} \gamma_{2}\left(\varepsilon_{1}, \varepsilon_{2}\right)=0$.

Including $\varepsilon_{4}$ produces $r=m=1, m-1=0$,

$\gamma_{0}\left(\varepsilon_{1}, \varepsilon_{2}, \varepsilon_{3}, \varepsilon_{4}\right)=1$

$\gamma_{1}\left(\varepsilon_{1}, \varepsilon_{2}, \varepsilon_{3}, \varepsilon_{4}\right)=\gamma_{1}\left(\varepsilon_{1}, \varepsilon_{2}, \varepsilon_{3}\right)+\varepsilon_{4} \gamma_{0}\left(\varepsilon_{1}, \varepsilon_{2}, \varepsilon_{3}\right)=.2905+.8450(1)=1.1356$.

$r=m=2, m-1=1$,

$\gamma_{2}\left(\varepsilon_{1}, \varepsilon_{2}, \varepsilon_{3}, \varepsilon_{4}\right)=\gamma_{2}\left(\varepsilon_{1}, \varepsilon_{2}, \varepsilon_{3}\right)+\varepsilon_{4} \gamma_{1}\left(\varepsilon_{1}, \varepsilon_{2}, \varepsilon_{3}\right)=0+.8450(.2905)=.2455$.

$r=m=3, m-1=2$,

$\gamma_{3}\left(\varepsilon_{1}, \varepsilon_{2}, \varepsilon_{3}, \varepsilon_{4}\right)=\gamma_{3}\left(\varepsilon_{1}, \varepsilon_{2}, \varepsilon_{3}\right)+\varepsilon_{4} \gamma_{2}\left(\varepsilon_{1}, \varepsilon_{2}, \varepsilon_{3}\right)=0+0=0$.

$r=m=4, m-1=3$,

$\gamma_{4}\left(\varepsilon_{1}, \varepsilon_{2}, \varepsilon_{3}, \varepsilon_{4}\right)=\gamma_{4}\left(\varepsilon_{1}, \varepsilon_{2}, \varepsilon_{3}\right)+\varepsilon_{4} \gamma_{3}\left(\varepsilon_{1}, \varepsilon_{2}, \varepsilon_{3}\right)=0+0=0$.

Including $\varepsilon_{5}$ produces $r=m=1, m-1=0$,

$$
\begin{aligned}
\gamma_{0}\left(\varepsilon_{1}, \varepsilon_{2}, \varepsilon_{3}, \varepsilon_{4}, \varepsilon_{5}\right) & =1=\frac{\partial^{2} \gamma_{2}}{\partial \varepsilon_{1} \partial \varepsilon_{2}}=\gamma_{0}^{(1,2)} \\
\gamma_{1}\left(\varepsilon_{1}, \varepsilon_{2}, \varepsilon_{3}, \varepsilon_{4}, \varepsilon_{5}\right) & =\gamma_{1}\left(\varepsilon_{1}, \varepsilon_{2}, \varepsilon_{3}, \varepsilon_{4}\right)+\varepsilon_{5} \gamma_{0}\left(\varepsilon_{1}, \varepsilon_{2}, \varepsilon_{3}, \varepsilon_{4}\right) \\
& =1.1356+1.8648(1)=3.0004=\frac{\partial^{2} \gamma_{3}}{\partial \varepsilon_{1} \partial \varepsilon_{2}}=\gamma_{1}^{(1,2)} .
\end{aligned}
$$

$r=m=2, m-1=1$,

$\gamma_{2}\left(\varepsilon_{1}, \varepsilon_{2}, \varepsilon_{3}, \varepsilon_{4}, \varepsilon_{5}\right)=\gamma_{2}\left(\varepsilon_{1}, \varepsilon_{2}, \varepsilon_{3}, \varepsilon_{4}\right)+\varepsilon_{5} \gamma_{1}\left(\varepsilon_{1}, \varepsilon_{2}, \varepsilon_{3}, \varepsilon_{4}\right)$

$$
=.2455+1.8648(1.1356)=2.3632=\frac{\partial^{2} \gamma_{4}}{\partial \varepsilon_{1} \partial \varepsilon_{2}}=\gamma_{2}^{(1,2)} \text {. }
$$

$r=m=3, m-1=2$,

$\gamma_{3}\left(\varepsilon_{1}, \varepsilon_{2}, \varepsilon_{3}, \varepsilon_{4}, \varepsilon_{5}\right)=\gamma_{3}\left(\varepsilon_{1}, \varepsilon_{2}, \varepsilon_{3}, \varepsilon_{4}\right)+\varepsilon_{5} \gamma_{2}\left(\varepsilon_{1}, \varepsilon_{2}, \varepsilon_{3}, \varepsilon_{4}\right)$

$$
=0+1.8648(.2455)=.4579=\frac{\partial^{2} \gamma_{5}}{\partial \varepsilon_{1} \partial \varepsilon_{2}}=\gamma_{3}^{(1,2)} .
$$

$r=m=4, m-1=3$,

$\gamma_{4}\left(\varepsilon_{1}, \varepsilon_{2}, \varepsilon_{3}, \varepsilon_{4}, \varepsilon_{5}\right)=\gamma_{4}\left(\varepsilon_{1}, \varepsilon_{2}, \varepsilon_{3}, \varepsilon_{4}\right)+\varepsilon_{5} \gamma_{3}\left(\varepsilon_{1}, \varepsilon_{2}, \varepsilon_{3}, \varepsilon_{4}\right)$ $=0+1.8648(0)=0$.

$r=m=5, m-1=4$,

$\gamma_{4}\left(\varepsilon_{1}, \varepsilon_{2}, \varepsilon_{3}, \varepsilon_{4}, \varepsilon_{5}\right)=\gamma_{4}\left(\varepsilon_{1}, \varepsilon_{2}, \varepsilon_{3}, \varepsilon_{4}\right)+\varepsilon_{5} \gamma_{3}\left(\varepsilon_{1}, \varepsilon_{2}, \varepsilon_{3}, \varepsilon_{4}\right)$ $=0+1.8648(0)=0$. 
This process generalizes to any pair of second derivatives. Simply set the particular pair of $\left(\varepsilon_{i}, \varepsilon_{k}\right)$ equal to 0 and execute the summation algorithm for $k$ items. Because the matrix of second derivatives is symmetric, the GAM subroutine is called $k(k-1) / 2$ times for a $k$-item test. Each call yields numericall values of the $\gamma_{(r-2)}^{(i . h)}$ for $r=1$, $2,3, \ldots, k$. This approach to computing derivatives of the ESFs is computationally intensive because the summation algorithm computations are performed for the full set of $k$ items each time. In addition, the entire summation algorithm is repeated $k$ times for the first derivatives and $k(k-1) / 2$ times for the second derivatives.

\section{Computing ESF Using the Grouping Property}

MacLane \& Birkhoff (1988) provided a proof of the grouping property of ESFs, and Verhelst \& Veldhuijzen (1991) used this property to construct an algorithm to compute the $\gamma_{r}$, as well as their first and second derivatives, in one process. However, for present purposes, using the grouping property only to compute the $\gamma_{r}$ will be illustrated [see Verhelst \& Veldhuijzen (1991) for the complete algorithm].

First, partition the $k$ item parameters into $p$ groups, each containing two item parameters. Next, the summation algorithm is used to compute the ESFs for each group of item parameters, which are called the building blocks. The grouping property is then used to compute the ESFS of a larger group formed by merging two of the groups. This hierarchical process is repeated until all $p$ groups are included and the ESFs of the full set of item parameters is obtained.

To illustrate this process, the parameter for an additional item was added to the set for LSAT-6. The value $\varepsilon_{6}$ $=1$ was selected so that certain computations could be related to earlier results and the set of six $\varepsilon$ parameters remained product-normalized. The set of six item parameters was partitioned into $p=3$ groups of size $m=2$ as follows:

$\left[\varepsilon_{1}, \varepsilon_{2}\right], \quad\left[\varepsilon_{3}, \varepsilon_{4}\right], \quad\left[\varepsilon_{5}, \varepsilon_{6}\right]$.

All arrangements of the item parameters into groups will produce the same final results. The summation algorithm was used to evaluate the $\gamma_{r}$ for each group. The building blocks are

Group $1 \gamma_{0}=1 \quad \gamma_{1}=\varepsilon_{1}+\varepsilon_{2}=4.1377 \quad \gamma_{2}=\varepsilon_{1} \varepsilon_{2}=2.1840$

Group $2 \gamma_{0}=1 \quad \gamma_{1}=\varepsilon_{3}+\varepsilon_{4}=1.1355 \quad \gamma_{2}=\varepsilon_{3} \varepsilon_{4}=.2455$

Group $3 \gamma_{0}=1 \quad \gamma_{1}=\varepsilon_{5}+\varepsilon_{6}=2.8648 \quad \gamma_{2}=\varepsilon_{5} \varepsilon_{6}=1.8648$.

Verhelst \& Veldhuijzen (1991) provided the following relationship:

$\gamma_{g}\left(k_{1}+k_{2}\right)=\sum_{i=a}^{b} \gamma_{i}\left(k_{1}\right) \gamma_{g-i}\left(k_{2}\right), g=0,1,2, \ldots,\left(k_{1}+k_{2}\right)$

where $a=\max \left(0, g-k_{2}\right), b=\min \left(g, k_{1}\right)$, and $k_{1}=k_{2}=2$.

To merge the first two groups, begin with

$r=0$,

then

$\gamma_{0}\left(k_{1}+k_{2}\right)=\sum_{i=0}^{0} \gamma_{0}\left(k_{1}\right) \gamma_{-1}\left(k_{2}\right)=1$

Because $\gamma_{-1}\left(k_{2}\right)=0$, this first result equals 1 by definition (Verhelst \& Veldhuijzen, 1991).

For $r=g=1$,

$$
\begin{array}{rlrl}
\gamma_{1}\left(k_{1}+k_{2}\right) i & =0 & \gamma_{0}\left(k_{1}\right) \gamma_{1}\left(k_{2}\right) & =(1)\left(\varepsilon_{3}+\varepsilon_{4}\right)=4.1337 \\
i & =1 & \gamma_{1}\left(k_{1}\right) \gamma_{0}\left(k_{2}\right) & =\left(\varepsilon_{1}+\varepsilon_{2}\right)(1)=1.1355 \\
\gamma_{1} & =\varepsilon_{1}+\varepsilon_{2}+\varepsilon_{3}+\varepsilon_{4}=5.2692 .
\end{array}
$$

For $r=g=2$, 


$$
\begin{aligned}
& \gamma_{2}\left(k_{1}+k_{2}\right) i=0 \quad \gamma_{0}\left(k_{1}\right) \gamma_{2}\left(k_{2}\right)=(1)\left(\varepsilon_{3} \varepsilon_{4}\right)=.2455 \\
& i=1 \quad \gamma_{1}\left(k_{1}\right) \gamma_{1}\left(k_{2}\right)=\left(\varepsilon_{1}+\varepsilon_{2}\right)\left(\varepsilon_{1}+\varepsilon_{2}\right)=4.6938 \\
& i=2 \quad \gamma_{2}\left(k_{1}\right) \gamma_{0}\left(k_{2}\right)=\left(\varepsilon_{1} \varepsilon_{2}\right)(1)=2.1840 \\
& \gamma_{2}=\varepsilon_{1} \varepsilon_{2}+\varepsilon_{1} \varepsilon_{3}+\varepsilon_{1} \varepsilon_{4}+\varepsilon_{2} \varepsilon_{3}+\varepsilon_{2} \varepsilon_{4}+\varepsilon_{3} \varepsilon_{4}=7.1233 \text {. }
\end{aligned}
$$

For $r=g=3$,

$$
\begin{aligned}
& \gamma_{3}\left(k_{1}+k_{2}\right) i=1 \quad \gamma_{1}\left(k_{1}\right) \gamma_{2}\left(k_{2}\right)=\left(\varepsilon_{1}+\varepsilon_{2}\right)\left(\varepsilon_{3} \varepsilon_{4}\right)=1.0148 \\
& i=2 \quad \gamma_{2}\left(k_{1}\right) \gamma_{1}\left(k_{2}\right)=\left(\varepsilon_{1} \varepsilon_{2}\right)\left(\varepsilon_{3}+\varepsilon_{4}\right)=2.9593 \\
& \gamma_{3}=\varepsilon_{1} \varepsilon_{3} \varepsilon_{4}+\varepsilon_{2} \varepsilon_{3} \varepsilon_{4}+\varepsilon_{1} \varepsilon_{2} \varepsilon_{3}+\varepsilon_{1} \varepsilon_{2} \varepsilon_{4}=3.4946 \text {. }
\end{aligned}
$$

For $r=g=4$,

$$
\begin{gathered}
\gamma_{4}\left(k_{1}+k_{2}\right) i=2 \quad \gamma_{2}\left(k_{1}\right) \gamma_{2}\left(k_{2}\right)=\left(\varepsilon_{1} \varepsilon_{2}\right)\left(\varepsilon_{3} \varepsilon_{4}\right)=(2.1840)(.2455) \\
\gamma_{4}=\varepsilon_{1} \varepsilon_{2} \varepsilon_{3} \varepsilon_{4}=.5361 .
\end{gathered}
$$

The numerical values of the $\gamma_{r}$ for the merged group of four items are the same as those obtained earlier when the fourth item parameter $\varepsilon_{4}$ was incorporated in the summation algorithm example. At this point, the $\gamma_{r}$ for one group of four items and one of two items are available. These two groups are merged to yield the ESFs for the full six-item test.

The building blocks now are the ESFs for the group of four items and those for the group of two items (Items 5 and 6); the numerical values of the ESFs were provided above. In Equation $94, k_{1}=4, k_{2}=2, a=$ $\max (0, g-2)$ and $b=\min (g, 4)$. Then, for $r=g=0$,

$$
\gamma_{0}\left(k_{1}+k_{2}\right)=\sum_{i=0}^{0} \gamma_{0}\left(k_{1}\right) \gamma_{-1}\left(k_{2}\right)=1
$$

For $r=g=1$,

$$
\begin{array}{rlrl}
\gamma_{1}\left(k_{1}+k_{2}\right) i & =0 & \gamma_{0}\left(k_{1}\right) \gamma_{1}\left(k_{2}\right) & =(1)(2.8648)=2.8648 \\
i & =1 & \gamma_{1}\left(k_{1}\right) \gamma_{0}\left(k_{2}\right) & =(5.2692)(1)=5.2692 \\
\gamma_{1} & =2.8648+5.2692=8.134 .
\end{array}
$$

For $r=g=2$,

$$
\begin{array}{rlrl}
\gamma_{2}\left(k_{1}+k_{2}\right) i & =0 & \gamma_{0}\left(k_{1}\right) \gamma_{2}\left(k_{2}\right)=(1)(1.8648)=1.8648 \\
i & =1 & \gamma_{1}\left(k_{1}\right) \gamma_{1}\left(k_{2}\right)=(5.2692)(2.8648)=15.0952 \\
i & =2 & \gamma_{2}\left(k_{1}\right) \gamma_{0}\left(k_{2}\right)=(7.1233)(1)=7.1233 \\
\gamma_{2} & =1.8648+15.0952+7.1233=24.0833
\end{array}
$$

For $r=g=3$,

$$
\begin{aligned}
& \gamma_{3}\left(k_{1}+k_{2}\right) i=0 \quad \gamma_{0}\left(k_{1}\right) \gamma_{3}\left(k_{2}\right)=0 \\
& i=1 \quad \gamma_{1}\left(k_{1}\right) \gamma_{2}\left(k_{2}\right)=(5.2692)(1.8648)=9.8260 \\
& i=2 \quad \gamma_{2}\left(k_{1}\right) \gamma_{1}\left(k_{2}\right)=(7.1233)(2.8648)=20.4068 \\
& i=3 \quad \gamma_{3}\left(k_{1}\right) \gamma_{0}\left(k_{2}\right)=(3.4946)(1)=3.4946 \\
& \gamma_{3}=9.8260+20.4068+3.4946=33.7274 \text {. }
\end{aligned}
$$

For $r=g=4$,

$$
\begin{array}{rr}
\gamma_{4}\left(k_{1}+k_{2}\right) i=0 & \gamma_{0}\left(k_{1}\right) \gamma_{4}\left(k_{2}\right)=0 \\
i=1 & \gamma_{1}\left(k_{1}\right) \gamma_{3}\left(k_{2}\right)=0
\end{array}
$$




$$
\begin{aligned}
& i=2 \quad \gamma_{2}\left(k_{1}\right) \gamma_{2}\left(k_{2}\right)=(7.1233)(1.8648)=13.2835 \\
& i=3 \quad \gamma_{3}\left(k_{1}\right) \gamma_{1}\left(k_{2}\right)=(3.4946)(2.8648)=10.0113 \\
& i=4 \quad \gamma_{4}\left(k_{1}\right) \gamma_{0}\left(k_{2}\right)=(.5361)(1)=.5361 \\
& \gamma_{4}=13.2835+10.0113+.5361=23.8310 \text {. }
\end{aligned}
$$

For $r=g=5$,

$$
\begin{aligned}
& \gamma_{5}\left(k_{1}+k_{2}\right) i=0 \quad \gamma_{0}\left(k_{1}\right) \gamma_{5}\left(k_{2}\right)=0 \\
& i=1 \quad \gamma_{1}\left(k_{1}\right) \gamma_{4}\left(k_{2}\right)=0 \\
& i=2 \quad \gamma_{2}\left(k_{1}\right) \gamma_{3}\left(k_{2}\right)=0 \\
& i=3 \quad \gamma_{3}\left(k_{1}\right) \gamma_{2}\left(k_{2}\right)=(3.4946)(1.8648)=6.5168 \\
& i=4 \quad \gamma_{4}\left(k_{1}\right) \gamma_{1}\left(k_{2}\right)=(.5361)(2.8648)=1.5358 \\
& i=5 \quad \gamma_{5}\left(k_{1}\right) \gamma_{0}\left(k_{2}\right)=0 \\
& \gamma_{s}=6.5168+1.5358=8.0526 \text {. }
\end{aligned}
$$

For $r=g=6$,

$$
\begin{aligned}
& \gamma_{6}\left(k_{1}+k_{2}\right) i=0 \quad \gamma_{0}\left(k_{1}\right) \gamma_{6}\left(k_{2}\right)=0 \\
& i=1 \quad \gamma_{1}\left(k_{1}\right) \gamma_{5}\left(k_{2}\right)=0 \\
& i=2 \quad \gamma_{2}\left(k_{1}\right) \gamma_{4}\left(k_{2}\right)=0 \\
& i=3 \quad \gamma_{3}\left(k_{3}\right) \gamma_{3}\left(k_{3}\right)=0 \\
& i=4 \quad \gamma_{4}\left(k_{1}\right) \gamma_{2}\left(k_{2}\right)=(.5361)(1.8648)=.9997 \\
& i=5 \quad \gamma_{5}\left(k_{1}\right) \gamma_{1}\left(k_{2}\right)=0 \\
& i=6 \quad \gamma_{6}\left(k_{1}\right) \gamma_{0}\left(k_{2}\right)=0 \\
& \gamma_{6}=.9997 \text {. }
\end{aligned}
$$

The subscripts on the $\gamma\left(k_{1}\right)$ vary from 0 to $r$, and those for $\gamma\left(k_{2}\right)$ vary from $r$ to 0 . Whenever the subscript of a $\gamma$ is greater than its group size, its value is 0 .

\section{Computing Derivatives of ESTS Using Liou's Extended Agmorithm}

Liou (1994) developed an algorithm for obtaining the first-and higher-order derivatives of ESFs that embodies the difference algorithm, the summation algorithm, and components of the grouping property of ESFS. Although Liou's algorithm can be used to obtain third-and higher-order derivatives, it is illustrated here for only the first and second derivatives of the ESFs.

To illustrate how first derivatives are computed, the six item parameter values from the grouping algorithm example are used because many of the needed numerical values are available in these earlier results. First, evaluate the $\gamma_{r}$ for the full set of $k=6$ items using the summation algorithm. In order to take the first derivatives of the $\gamma_{r}$ for the full set of 6 items with respect to $\varepsilon_{6}$, a group will be defined that only contains this $\varepsilon$ parameter. Equation 13 of the difference algorithm is used recursively in the low-to-high direction in the following manner:

$$
\begin{aligned}
\gamma_{0}^{(6)} & =1 \\
\gamma_{1}^{(6)} & =\gamma_{1}-\varepsilon_{6} \gamma_{0}^{(6)} \\
& =8.1340-(1)(1)=7.1340 .
\end{aligned}
$$

As first observed for the summation algorithm, 
$\gamma_{1}^{(6)}=\frac{\partial \gamma_{2}}{\partial \varepsilon_{6}}$

is the derivative of the next higher-order ESF involving all $k$ items with respect to $\varepsilon_{6}$, and

$\frac{\partial \gamma_{2}}{\partial \varepsilon_{6}}=\gamma_{1}\left(\varepsilon_{1}, \varepsilon_{2}, \varepsilon_{3}, \varepsilon_{4}, \varepsilon_{5}\right)$

is the first-order ESF for the remaining 5 items. Then

$$
\begin{aligned}
\gamma_{2}^{(6)} & =\gamma_{2}-\varepsilon_{6} \gamma_{1}^{(6)} \\
& =24.0833-(1)(7.1340)=16.9493 .
\end{aligned}
$$

The $\gamma_{1}^{(6)}$ term was available from the previous computation, and when multiplied by $\varepsilon_{6}$ yields the term to be subtracted from the next higher-order ESF. Thus, as each first derivative is computed it is used in Equation 13 to compute the next higher-order first derivative. Continuing the sequence,

$$
\begin{aligned}
\gamma_{3}^{(6)} & =\gamma_{3}-\varepsilon_{6} \gamma_{2}^{(6)} \\
& =33.7274-(1)(16.9493)=16.7781 \\
\gamma_{4}^{(6)} & =\gamma_{4}-\varepsilon_{6} \gamma_{3}^{(6)} \\
& =23.8310-(1)(16.7781)=7.0529 \\
\gamma_{5}^{(6)} & =\gamma_{5}-\varepsilon_{6} \gamma_{4}^{(6)} \\
& =8.0526-(1)(7.0529)=.9997 .
\end{aligned}
$$

That these first derivatives are the same as the $\gamma_{r}$ for the five items of the LSAT- 6 data can be verified by examining Table 3 . The advantage of Liou's extended algorithm is that it avoids the computation of a long series of intermediate ESFS to reach those needed.

In order to obtain the second derivatives using Liou's algorithm, the $k$ item parameters are partitioned again. For purposes of this example, the second derivatives will be taken with respect to $\varepsilon_{5}$ and $\varepsilon_{6}$, which will constitute the group $\left(k_{1}=2\right)$. Liou (1994) provided the following equation for obtaining the second derivatives:

$$
\gamma_{r}^{(i, j)}=\gamma_{r}-\left[\gamma_{1}\left(k_{1}\right) \gamma_{r-1}^{(i, j)}+\gamma_{2}\left(k_{1}\right) \gamma_{r-2}^{(i, j)}\right]
$$

where $\gamma_{r}$ is the ESF of order $r$ for the complete set of $k$ items and $\gamma_{1}\left(k_{1}\right)$ and $\gamma_{2}\left(k_{1}\right)$ are for the group of size two.

Implementing Liou's extended algorithm requires the initial computation of the $\gamma_{r}$ for the full set of $k$ items and for the group of two items using the summation algorithm. The numerical values for the full set were provided above in the example of the grouping algorithm. Those for the group of two are $\gamma_{0}\left(k_{1}\right)=1$, $\gamma_{1}\left(k_{1}\right)=\varepsilon_{5}+\varepsilon_{6}=1.8648+1.0000=2.8648$, and $\gamma_{2}\left(k_{1}\right)=\varepsilon_{5} \varepsilon_{6}=1.8648$.

Given these initial values, the algorithm proceeds as follows:

$$
\begin{aligned}
\gamma_{-1}^{(5,6)} & =0 \\
\gamma_{0}^{(5,6)} & =1 \\
\gamma_{1}^{(5,6)} & =\gamma_{1}-\left[\gamma_{1}\left(k_{1}\right) \gamma_{0}^{(5.6)}+\gamma_{2}\left(k_{1}\right) \gamma_{-1}^{(5,6)}\right] \\
& =8.1340-[(2.8648)(1)+(1.8648)(0)]=5.2692 \\
\gamma_{2}^{(5,6)} & =\gamma_{2}-\left[\gamma_{1}\left(k_{1}\right) \gamma_{1}^{(5,6)}+\gamma_{2}\left(k_{1}\right) \gamma_{0}^{(5.6)}\right] \\
& =24.0833-[(2.8648)(5.2692)+(1.8648)(1)]=7.1233 \\
\gamma_{3}^{(5,6)} & =\gamma_{3}-\left[\gamma_{1}\left(k_{1}\right) \gamma_{2}^{(5,6)}+\gamma_{2}\left(k_{1}\right) \gamma_{1}^{(5,6)}\right] \\
& =33.7274-[(2.8648)(7.1233)+(1.8648)(5.2692)]=3.4946
\end{aligned}
$$




$$
\begin{aligned}
\gamma_{4}^{(5,6)} & =\gamma_{4}-\left[\gamma_{1}\left(k_{1}\right) \gamma_{3}^{(5,6)}+\gamma_{2}\left(k_{1}\right) \gamma_{2}^{(5,6)}\right] \\
& =23.8310-[(2.8648)(3.4946)+(1.8648)(7.1233)]=.5361 .
\end{aligned}
$$

Because

$$
\gamma_{r}^{(5,6)}=\frac{\partial \gamma_{r}}{\partial \varepsilon_{5} \partial \varepsilon_{6}}=\gamma_{r}\left(\varepsilon_{1}, \varepsilon_{2}, \varepsilon_{3}, \varepsilon_{4}\right)
$$

these second derivatives are the same as the $\gamma_{r}$ when $k=4$ and only the first four items are involved. This can be verified by examining the numerical results presented above for the summation algorithm example when the fourth parameter $\varepsilon_{4}$ was added. These results show that the second derivatives of $\gamma_{r}$ for $k$ items can be computed by grouping the items such that the first group contains the item parameters with which the derivatives are to be taken, and the second group the remaining item parameters. The $\gamma_{r}$ for the second group will be those for the derivatives of interest. Because CMLE needs the $\gamma_{r}$ for the full set of $k$ items, Liou's algorithm produces the second derivatives needed in the Newton-Raphson procedure much more efficiently than the summation algorithm for a group of $k-2$ items. In practice, Liou's algorithm is implemented using a low-to-high part and a high-to-low part, as Fischer (1974) did, to prevent the rounding errors inherent in the difference algorithm from propagating into the higher-order ESFs when $r>k / 2$.

\section{Comparison of the Algorithms}

The algorithms presented here exploit the fact that an ESF of order $r$ based on some subset of size $k-v$ $\varepsilon$ parameters is the same as the $v$ th derivative of an ESF of order $r$ for the complete set of $k$ item parameters. Inspection of earlier calculations for the various algorithms reveals many instances in which the relationships between the ESFS and their derivatives are being used-for example, the derivatives presented in Table 4 for the Verhelst et al. (1984) reformulation of the summation algorithm. In the difference and summation algorithms, $v=1$. The difference algorithm obtains the $\gamma_{r}^{(i)}$ by using this relationship to remove the appropriate terms contained in a subset of $k-1$ item parameters from the corresponding $\gamma_{r}$ based on the full set of $k$ item parameters. Liou's extended algorithm uses the relationship in a similar manner. The summation algorithm uses it to add the appropriate terms to the $\gamma_{r}$ from a smaller set to the $\gamma_{r}$ for the larger set. However, it does this recursively within a subset of items as the number of items in as subset, $m$, increases by 1 until $m=k$.

The computations for each of the algorithms are relatively simple, but their number grows dramatically as the size of a test is increased. Increasingly large tests $(k>50)$ present two problems that plague the algorithms: (1) excessive computer time, which relates to efficiency or cost; and (2) numerical accuracy, which limits the capacity of the algorithms. Comparing the performance of the algorithms on these two issues exposes their similarities and differences.

\section{Conmpurer Time}

The computer time problem arises from the fact that a given $\gamma_{r}$ involves the sum of $\left(\begin{array}{l}k \\ r\end{array}\right)$ terms, each of which involves the product of $r \varepsilon$ parameters. For example, the difference algorithm requires $2 k^{2}$ multiplications/divisions and $2 k^{2}$ subtractions/additions to compute a full set of $\gamma_{r}$ for a $k$-item test; the summation algorithm requires $k(k-1) / 2$ multiplications and $k(k-1) / 2+(k+1)$ additions (Gustafsson, 1980). [A reviewer indicated that the total count of multiplications for one run of the difference algorithm is $2 k^{2}$ and that the total count for one run of the combination algorithm is approximately $k(k+1) / 2+4 k(k-1)$. Thus, the latter should be more computationally demanding than the difference algorithm alone.]

Gustafsson indicated that, relative to the difference algorithm, the summation algorithm is slower by a factor of $k / 4$. Verhelst \& Veldhuijzen (1991) measured the computer time demand in terms of cost units, 
where a unit consisted of one multiplication and one addition. The cost of the summation algorithm for computing the ESFs and their first- and second-order derivatives for a $k$-item test was $1 / 4 k^{4}-1 / 2 k^{3}+5 / 4 k^{2}$. Formann (1986) stated that the number of operations needed for the evaluation of all $k(k-1) / 2$ secondorder derivatives increases as a function of $k^{3}$ for the difference algorithm and a function of $k^{4}$ for the summation algorithm. The fact that CMLE is iterative and the complete set of computations are repeated using new values of the $\varepsilon_{i}$ until a convergence criterion is met compounds the computer time problem.

Surprisingly little empirical evidence on the amount of computer time required is available. Liou (1994) provided some empirical run times for the calculation of derivatives using her extended algorithm and the summation algorithm. Liou's computer programs were written in FORTRAN using double precision (REAL*8) arithmetic on a VAX 8350 minicomputer and a SUN SPARC-10 work station. She found that for 60 items, the summation algorithm required approximately 4.2 seconds on the VAX and 1 seconds on the SUN workstation to compute the first derivatives of the ESFs. In contrast, Liou's extended algorithm used approximately 1 seconds on the VAX and less than 1 millisecond on the SUN workstation. Liou's findings suggest the obvious conclusion that the "power" of the computer has a large impact on the computer time used, and that her extended algorithm is very efficient compared to the summation algorithm for computing derivatives.

Similarly, Verhelst \& Veldhuijzen (1991) reported an $80 \%$ and $90 \%$ reduction in computer time for their new algorithm relative to that of the summation algorithm for computing first- and second-order derivatives, respectively. Because a considerable amount of test calibration takes place on personal computers, it would be useful to have additional comparative data for these algorithms using commonly available personal computers.

\section{Numerical Accuracy}

The second problem area is that of numerical accuracy, which directly affects the size of the test that can be calibrated using CMLE. Although the capacity of all the algorithms is limited by the numerical accuracy factor, it is a major problem for the difference algorithm because the basic recursion (Equation 13) involves subtraction. When the order of the ESF approaches $k / 2$, the difference between two very large numbers is taken and rounding errors in these two numbers can have a major impact. Numerical accuracy is also adversely impacted by the ratio of $\varepsilon_{\max }$ to $\varepsilon_{\min }$, the ratio of the largest values of $\varepsilon_{i}$ to the smallest value of $\varepsilon_{i}$ in its set of items (Gustafsson, 1980; Verhelst et al., 1984). When this ratio is large, the terms in the ESFs involve elements of mixed magnitudes that lead to rounding errors. Verhelst et al. (1984) provided an excellent exposition of the numerical problems inherent in the evaluation of ESFS.

Gustafsson (1980) proposed the combination algorithm to reduce the impact of rounding errors. The summation algorithm is used to compute the $\gamma_{r}$, and Equation 13 is then used to compute the first-order derivatives of the ESFS. At this point, the $\gamma_{r}^{(i)}$ are available and the $\gamma_{r}$ are computed using Equation 14. Fischer's ratio test then can be used to compare the $\gamma_{r}$ computed using the first derivatives with the corresponding $\gamma_{r}$ based on the summation algorithm. When the test criterion for a given $\gamma_{r}$ is exceeded, the first derivatives are recomputed using the summation algorithm and the $\varepsilon_{i}=0$ technique, and a new value for $\gamma_{r}$ is obtained. Gustafsson (1980) reported that when no recomputation was necessary, the combination algorithm was slightly faster than the difference algorithm and that it enabled tests of up to $80-100$ items to be analyzed. However, no empirical evidence to support this claim was provided.

The current version of the PML computer program (Gustafsson, 1990) provides the user the option of using the difference, summation, or combination algorithm to compute the ESFS needed in CMLE of the item parameters under the Rasch model. However, the PML program manual (Molenaar, 1990) places an upper limit on the number of items at 60 for all three algorithms.

Fischer \& Ponocny $(1994,1995)$ developed an improved version of the combination algorithm that is both fast and accurate. The $\gamma_{r}$ for the full set of $k$ items are computed using the summation algorithm, and the $\gamma_{r-1}^{(i)}$ 
for $r=1,2, \ldots, k$ are computed for each item separately using the difference algorithm. The "bottom-up" approach is used for $r=1,2, \ldots, r^{\prime}$, and the "top-down" approach for $r=k-1, k-2, \ldots, r$ '. The index value $r^{\prime}$ is determined for each item (see Fischer \& Ponocny, 1995, eq. 19.24), and an accuracy check is made at $r$ $=r^{\prime}$ for each item. If the result is unsatisfactory, the respective $\gamma_{r-1}^{(i)}$ for $r=1,2, \ldots, k-1$ are recomputed for that item using the summation algorithm. Fischer (personal communication, November, 1995) reported that using this algorithm in the LLTM computer program (Seliger \& Fischer, 1994) allowed the Rasch model item difficulty parameters of a 150 -item test to be estimated in 32 seconds on a $486 \mathrm{Dx} 100 \mathrm{mhz}$ computer, indicating that the algorithm is very fast.

\section{Condusions}

Past computational difficulties in computing ESFs and their derivatives have limited the use of CMLE of irem parameters under the Rasch model. For example, Wright \& Douglas (1977) were able to estimate parameters for only 12-15 items before numerical instability set in. However, the introduction of efficient algorithms and the availability of high-speed personal computers have increased the attractiveness of this method. Fischer's (1974) bidirectional version of the difference algorithm increased the capacity to approximately 40 items, and Gustafsson's (1980) combination algorithm allowed 80-100 items to be calibrated. Verhelst \& Veldhuijzen (1991) reported considerable savings in computer time by using the summation algorithm and the grouping property of ESFs to calibrate data for 144 parameters, and Fischer \& Ponocny (1994, p. 183) provided an improved combination algorithm that allows 150 items to be calibrated.

Perhaps the most important consequence of these advances is that the newer algorithms for computing and evaluating ESFs have sufficient capacity for the CMLE procedure to calibrate typical educational and psychological instruments. However, the problems of computer time and numerical accuracy still have not been completely resolved.

\section{References}

Andersen, E. B. (1970). Asymptotic properties of conditional maximum likelihood estimators. The Journal of the Royal Statistical Society, 32, (Series B), 283-301.

Andersen, $\mathbb{E}$. B. (1972). The numerical solution of a set of conditional estimation equations. The Journal of the Royal Statistical Society, 34, (Series B), 42-54.

Bock, R. D., \& Lieberman, M. (1970). Fitting a response model for $n$ dichotomously scored items. Psychometrika, 35, 179-197.

Fischer, G. H. (1973). The linear logistic test model as an instrument in educational research. Acta Psychological, 37, 359-374.

Fischer, G. H. (1974). Einfihhrung in die Theorie psychologischer Tests Introduction to the theory of psychological tests]. Bern: Huber.

Fischer, G. H. (1995). The linear logistic test model. In G. H. Fischer \& I. W. Molenaar (Eds.), Rasch models: Foundations, recent developments, and applications (chap. 8, pp. 131-155). New York: Springer-Verlag.

Fischer, G. H., \& Allerup, P. (1968). Rechentchnische fragen zu Raschs eindimensionalem modell [An inquiry into computational rechniques for the Rasch model]. In G. H. Fischer (Ed.), Psychologische Testheorie (chap. 10). Bern: Huber.

Fischer, G.H., \& Formann, A. K. (1972). An algorithm and a FORTRAN program for estimating the item parameters of the linear logistic test model (Research Bulletin No. 11). Vienna: Psychologisches Institute der Universitat Wien.

Fischer, G. H., \& Ponocny, I. (1994). An extension of the partial credit model with an application to the measurement of change. Psychometrika, 59, 177-192.

Fischer, G. H., \& Ponocny, 1. (1995). Extending rating scale and partial credit models for assessing change. In G. H. Fischer \& I. W. Molenaar (Eds.), Rasch models: Foundations, recent developments, and applications (chap. 19, pp. 361-364). New York: SpringerVerlag.

Fischer, G. H., \& Wild, B. (1988). Program LLTM [Computer program.] Vienna, Austria: Psychologisches Institute der Universitat Wien.

Formann, A. K. (1986). A note on the computation of the second-order derivatives of the elementary symmetric functions in the Rasch model. Psychometrika, 51. 335-339.

Gustafsson, J.-E. (1977). The Rasch model for dichotomous items: Theory, applications and a computer program. Göteborg, Sweden: The Institute of Education, University of Göteborg. (ERIC Document Reproduction Service No. ED 154018) 
Gustafsson, J.-E. (1980). A solution of the conditional estimation problem for long tests in the Rasch model for dichotomous items. Edlecational and Psychological Measurement, 40, 327-385.

Gustafsson, J.-E. (1990). PML: PC version [Computer program]. Groningen, The Netherlands: iec ProGAMMA.

Jansen, P. G. (1984). Computing the second-order derivatives of the symmetric functions in the Rasch model. Kwantitatieve Methoden, 13, 131-147.

Liou, M. (1994). More on the computation of higherorder derivatives of the elementary symmetric functions in the Rasch model. Applied Psychological Measurement, 18, 53-62.

Liou, M., \& Chang, C.-H. (1992). Constructing the exact significance levell for a person fit statistic. Psychometrika, 57, 169-181.

MacLane, S., \& Birkhoff, G. (1988). Algebra (3rd ed.) New York: Chelsea.

Molenaar, I. W. (1990). PML: User's manual (PC version) [Computer program manual]. Groningen, The Netherlands: iec ProGAMMA.

Rasch, G. (1960/80). Probabilistic models for some intelligence and attainment tests. (Copenhagen, Danish Institute for Educational Research). Expanded edition (1980), with foreword and afterword by B. D. Wright. Chicago: The University of Chicago Press.

Rasch, G. (1961). On general laws and the meaning of measurement in psychology. In J. Neyman (Ed.), Pro- ceedings of the Fourth Berkeley Symposium on Mathematical Statistics, 4, 321-334. Berkeley: University of Califomia Press.

Seliger, E., \& Fischer, G. H. (1994). Program description LRSMG, LRSM, LTTM, LPCM, with applications to scale analysis and measuring change. Vienna, Austria: Psychologisches Institute der Universitat Wien.

Thissen, D. (1982). Marginal maximum likelihood estimation for the one-parameter logistic model. Psychometrika, 47, 175-186.

Verhelst, N. D., Glas, C. A. W., \& van der Sluis, A. (1984). Estimation problems in the Rasch model: The basic symmetric functions. Computational Statistics Quarterly, 1, 245-262.

Verhelst, N. D., \& Veldhuijzen, N. H. (1991). A new algorithm for computing elementary symmetric functions and their first and second derivatives (Measurement and Research Department Reports 91-1). Arnhem, The Netherlands: Netherlands Central Bureau of Statistics.

Wright, B. D., \& Douglas, G. A. (1977). Conditional versus unconditional procedures for sample-free analysis. Educational and Psychological Measurement, 37, 573-586.

\section{Anthor"s Address}

Send requests for reprints or further information to Frank B. Baker, 1025 W. Johnson Street, Department of Educational Psychology, University of Wisconsin, Madison WI 53706, U.S.A. Email: fbbaker@ facstaff.wisc.edu. 